Article

\title{
stim $2 b$ Knockout Induces Hyperactivity and Susceptibility to Seizures in Zebrafish Larvae
}

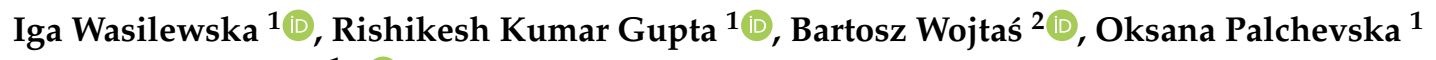 \\ and Jacek Kuźnicki ${ }^{1, *(1)}$ \\ 1 International Institute of Molecular and Cell Biology, 4 Ks. Trojdena Street, 02-109 Warsaw, Poland; \\ iwasilewska@iimcb.gov.pl (I.W.); rkgupta@iimcb.gov.pl (R.K.G.); opalchevska@iimcb.gov.pl (O.P.) \\ 2 Nencki Institute of Experimental Biology, Polish Academy of Sciences, 3 Pasteur Street, \\ 02-093 Warsaw, Poland; b.wojtas@nencki.edu.pl \\ * Correspondence: jacek.kuznicki@iimcb.gov.pl
}

Received: 30 April 2020; Accepted: 20 May 2020; Published: 21 May 2020

\begin{abstract}
In neurons, stromal interaction molecule (STIM) proteins regulate store-operated $\mathrm{Ca}^{2+}$ entry (SOCE) and are involved in calcium signaling pathways. However, STIM activity in neurological diseases is unclear and should be clarified by studies that are performed in vivo rather than in cultured cells in vitro. The present study investigated the role of neuronal Stim $2 \mathrm{~b}$ protein in zebrafish. We generated $\operatorname{stim} 2 b$ knockout zebrafish, which were fertile and had a regular lifespan. Using various behavioral tests, we found that stim $2 b^{-/}$zebrafish larvae were hyperactive compared with wild-type fish. The mutants exhibited increases in mobility and thigmotaxis and disruptions of phototaxis. They were also more sensitive to pentylenetetrazol and glutamate treatments. Using lightsheet microscopy, a higher average oscillation frequency and higher average amplitude of neuronal $\mathrm{Ca}^{2+}$ oscillations were observed in stim $2 b^{-/}$larvae. RNA sequencing detected upregulation of the annexin $3 a$ and gpr39 genes and downregulation of the rrm2, neuroguidin, and homer2 genes. The latter gene encodes a protein that is involved in several processes that are involved in $\mathrm{Ca}^{2+}$ homeostasis in neurons, including metabotropic glutamate receptors. We propose that Stim $2 \mathrm{~b}$ deficiency in neurons dysregulates SOCE and triggers changes in gene expression, thereby causing abnormal behavior, such as hyperactivity and susceptibility to seizures.
\end{abstract}

Keywords: Stim2b; zebrafish; behavior; in vivo imaging; calcium; GCaMP5G; seizures

\section{Introduction}

Stromal interaction molecule (STIM) is a calcium $\left(\mathrm{Ca}^{2+}\right)$-sensing protein that is localized in the endoplasmic reticulum (ER) membrane [1]. In the luminal part, it contains a highly conserved $\mathrm{Ca}^{2+}$-binding EF-hand domain. The cytosolic part contains the CRAC activation domain (CAD; also known as the STIM1 Orai1 activating region [SOAR]), which is responsible for interactions with plasma membrane (PM) $\mathrm{Ca}^{2+}$ channels and communication with calmodulin [2]. Mammals express two paralogues of STIM: Stim1 and Stim2, while in fish, an additional genome duplication resulted in the appearance of two additional genes that encode Stim proteins: stim $1 a$, stim $1 b$, stim2a, and stim $2 b[3,4]$. Both STIM1 and STIM2 were found to be ubiquitously expressed with varying STIM1-to-STIM2 ratios, depending on the specific tissue. In mice, STIM1 expression dominates over STIM2 expression. One exception is in the brain, mostly in the hippocampus and cortex, where higher amounts of STIM2 mRNA and protein expression have been observed [5-7]. In the human brain, the expression of both STIM1 and STIM2 was highest in the cortex, caudate, and hippocampus [6]. The main function of STIMs is the regulation of store-operated $\mathrm{Ca}^{2+}$ entry (SOCE), which enables the influx of $\mathrm{Ca}^{2+}$ from the external milieu into the cell under conditions of ER depletion. Upon interactions with STIM proteins, 
$\mathrm{Ca}^{2+}$ enters the cell via Orai channels that are highly selective for these ions or via less selective transient receptor potential channels [8]. The ER is then refilled with $\mathrm{Ca}^{2+}$ by the sarcoendoplasmic reticulum $\mathrm{Ca}^{2+}$ adenosine triphosophatase (SERCA) pump. The current model of SOCE considers different properties of STIM isoforms and proposes a biphasic response. Small decreases in $\mathrm{Ca}^{2+}$ levels in the ER are detected by STIM2, which is more sensitive to changes in $\mathrm{Ca}^{2+}$ levels and induces small but prolonged SOCE. STIM1 is activated by larger decreases in ER $\mathrm{Ca}^{2+}$ levels and triggers robust and rapid $\mathrm{Ca}^{2+}$ influx [2,9]. Notably, refilling the ER with $\mathrm{Ca}^{2+}$ is not the only consequence of SOCE. $\mathrm{Ca}^{2+}$ that enters the cell is involved in different signaling pathways. One of the most studied pathways is the activation of gene transcription via the calcineurin-nuclear factor of activated T-cells (NFAT) pathway (reviewed in $[9,10]$ ).

Research on the role of SOCE in neurons was relatively neglected for quite a long time. The regulation of $\mathrm{Ca}^{2+}$ signaling in these cells is very complex and requires tight spatial and temporal control because these ions are crucial for various neuronal processes, including neurotransmitter release, synaptic plasticity, neurogenesis, and gene transcription. Thus, neurons developed highly complicated $\mathrm{Ca}^{2+}$ machinery [11]. The main pathways that enable the influx of $\mathrm{Ca}^{2+}$ into these cells involve $\mathrm{Ca}^{2+}$-permeable voltage-operated $\mathrm{Ca}^{2+}$ channels (VOCCs) or receptor-operated channels (ROCs), such as glutamate-sensitive $N$-methyl-D-aspartate receptors (NMDARs). Nevertheless, SOCE has also been observed in neurons (reviewed in $[9,10,12]$ ). Our group demonstrated that the overexpression of STIM1 together with Orai1 increased $\mathrm{Ca}^{2+}$ entry that was induced by ER depletion with the potent SERCA inhibitor thapsigargin in cortical rat neurons, whereas the overproduction of STIM2 and Orai1 increased constitutive $\mathrm{Ca}^{2+}$ entry. Stronger interactions between STIM2 and Orai1 were observed in the absence of extracellular $\mathrm{Ca}^{2+}[13,14]$. Similar observations were made by others using SOCE inhibitors or knocking down the main SOCE components $[15,16]$. SOCE has been implicated in various neuronal functions, such as neuronal plasticity [15,17], the expression and activity of PM receptors [18,19], neuronal excitability [6,20], and the regulation of gene expression [16]. Disruptions of SOCE have been implicated in several neurodegenerative diseases, including Alzheimer's disease [17,21], Huntington's disease [22,23], and epilepsy [6] (reviewed in [10,24]).

Notably, STIM2 appears to be crucial in regulating $\mathrm{Ca}^{2+}$ homeostasis in neurons. Reductions of both SOCE and basal $\mathrm{Ca}^{2+}$ levels were observed in cortical neurons that were isolated from Stim2 $2^{-/-}$ mice [25]. STIM2 was also essential for SOCE in hippocampal neurons [17]. Descriptions of Stim2 ${ }^{-/}$ mice have not been fully consistent. Oh-Hora et al. reported early lethality in Stim2 ${ }^{-/-}$mice at 4-5 weeks of age [26]. Similarly, Berna-Erro et al. observed a lower life expectancy (i.e., 8 weeks) in Stim $2^{-/-}$ mice [25]. The mechanism of death in Stim2 $2^{-/-}$mice remains unknown. However, another study showed no changes in mortality in Stim $2^{-/-}$mice [27]. Notably, however, Stim knockout in this work was restricted only to the forebrain. Despite a growing number of studies, the role of STIM2 in shaping behavior and neuronal activity remains elusive. In addition to the complexity of various processes that affect $\mathrm{Ca}^{2+}$ homeostasis in neurons, early lethality in mice that are devoid of STIM2 makes examining these aspects extremely challenging. The vast majority of studies have been performed in cell cultures, with a lack of such investigations in live organisms.

Zebrafish are a powerful model that allows combinations of behavioral tests, the in vivo imaging of neuronal activity, and molecular analyses. Studying zebrafish behavior provides vast possibilities even during the larval stage (reviewed in $[28,29]$ ). Assays that enable assessments of anxiety levels, such as thigmotaxis $[30,31]$ and phototaxis $[32,33]$, have been well established in this model. Zebrafish are also often used for drug screening, and several neuroactive compounds have been tested in this animal $[28,34]$. Among these compounds are pro-convulsant drugs, such as pentylenetetrazol (PTZ), that induce robust changes in larval behavior and neuronal activity [35].

In the present study, we took advantage of genome duplication that occurs in zebrafish. Through this process, zebrafish possess two genes that encode Stim 2 proteins: $\operatorname{sim} 2 a$ and $\operatorname{stim} 2 b[3,4]$. These genes share $\sim 54 \%$ sequence identity (according to Ensembl release 99 [36]). The stim $2 b$ sequence is more similar to its human orthologue ( $\sim 59 \%$ sequence identity) than the stim $2 a$ sequence $(\sim 49 \%$ 
sequence identity). Our group showed the presence of transcripts for both stim 2 genes and genes that encode other SOCE components in the adult zebrafish brain and head of zebrafish larvae [4]. Moreover, the mechanism of SOCE appears to be conserved in zebrafish and plays an important role in the development of this organism. The inhibition of this process disrupts cytokinesis in zebrafish embryos [37]. The downregulation of Stim1 in zebrafish resulted in disruptions of axonal growth and motor function [38]. Mutations of the stim1 gene in zebrafish were also shown to affect the pigmentation process [39]. In the present study, we further characterized the phenotype of $\operatorname{sim} 2 b^{-/}$zebrafish.

To unveil the function of Stim $2 b$, we investigated the effects of Stim $2 b$ depletion on neuronal $\mathrm{Ca}^{2+}$ homeostasis and behavior. We investigated changes in gene expression that were induced by $s t i m 2 b$ knockout. We performed an analysis of mutant locomotor activity using several behavioral tests. We also investigated the effects of treatment with drugs that modulate neuronal signaling on mobility. The behavioral analyses were supplemented with analyses of neuronal $\mathrm{Ca}^{2+}$ signaling in live zebrafish.

\section{Materials and Methods}

\subsection{Animal Maintenance}

Wild-type (WT; AB line), stim $2 b^{-/}$, and $\operatorname{Tg}(H u C: G C a M P 5 G)$ [40] zebrafish were used in the study. All of the animals were maintained according to previously described methods [41] in the Zebrafish Core Facility that is a licensed breeding and research facility (PL14656251, registry of the District Veterinary Inspectorate in Warsaw; 064 and 051, registry of the Ministry of Science and Higher Education) at the International Institute of Molecular and Cell Biology in Warsaw. All of the experiments with larvae and adult fish were performed in accordance with the European Communities Council Directive (63/2010/EEC). Adult zebrafish and larvae were kept in E3 medium (2.48 $\mathrm{mM} \mathrm{NaCl}$, $0.09 \mathrm{mM} \mathrm{KCl}, 0.164 \mathrm{mM} \mathrm{CaCl}_{2} \cdot 2 \mathrm{H}_{2} \mathrm{O}$, and $\left.0.428 \mathrm{mM} \mathrm{MgCl}_{2} \cdot 6 \mathrm{H}_{2} \mathrm{O}\right)$ at $28.5^{\circ} \mathrm{C}$. Larvae were kept in a Petri dish ( 50 larvae/dish) in an incubator under a $14 \mathrm{~h} / 10 \mathrm{~h}$ light/dark cycle. The stages of fish development were defined as hours postfertilization (hpf) and days postfertilization (dpf).

\subsection{Generation and Genotyping of stim $2 b^{-/-}$Mutant Zebrafish}

The clusters of regularly interspaced short palindromic repeats (CRISPR)/Cas9 system was applied to generate $\operatorname{stim} 2 b^{-/}$zebrafish. CRISPR/Cas9 target sequences were selected using the web-based tool "CHOP CHOP" (https://chopchop.rc.fas.harvard.edu/; accessed September 15, 2014). The CRISPR/Cas9 target site (GGACCAGCACATCACGGTGG AGG) was localized in the exon 3 of stim $2 b$ (ENSDARG00000001776/ENDART00000012089). The templates for CRISPR guide RNA (gRNA) were prepared by polymerase chain reaction (PCR). The PCR fragments were column-purified using the QIAquick PCR Purification Kit (Qiagen, catalog no. 28106, Hilden, Germany) and transcribed using the mMESSAGE mMACHINE T7 Transcription Kit (Life Technologies, catalog no. AM1344M, Carlsbad, CA, USA) or the MEGAshortscript T7 Transcription Kit (Life Technologies, catalog no. AM1354, Carlsbad, CA, USA). The RNA was then column-purified with the miRNeasy Mini kit (Qiagen, catalog no. 217004, Hilden, Germany), and its quality was checked using an Agilent RNA 6000 Nano Chip (Agilent 2100 bioanalyzer, Santa Clara, CA, USA). A mixture that contained gRNA and Cas9 RNA ( $300 \mathrm{ng} / \mu \mathrm{L} ; 1: 1$ ) was injected into one-cell-stage embryos (in a volume of $1 \mu \mathrm{L}$ ). The offspring of stim $2 b$-targeted fish were screened using DNA sequencing of the targeted region for the presence of indel mutations. Fish with the insertion of 4 nucleotides were selected as P0 founders. F2 fish were outcrossed with the $\mathrm{AB}$ zebrafish line, and their offspring were in-crossed to generate homozygous mutants. The behavioral and real-time PCR (RT-PCR) and RNA sequencing (RNAseq) experiments were performed using the offspring of these fish (or fish from subsequent generations). For the $\mathrm{Ca}^{2+}$ imaging experiments, stim $2 b^{-/-}$fish were outcrossed with $\operatorname{Tg}(H u C: G C a M P 5 G)$ fish.

The offspring of $\mathrm{P} 0$ stim $2 b^{-/}$founder fish were genotyped using high-resolution melting (HRM) analysis. Genetic material was obtained from the caudal fin of an adult fish, and the targeted region of stim $2 b$ was amplified using LightCycler 480 High-Resolution Melting Master (Roche, catalog 
no. 04909631001, Basel, Switzerland) and the LightCycler 96 System (Roche, Basel, Switzerland) or using Precision Melt Supermix (Bio-Rad, catalog no. 1725112, Hercules, CA) and the CFX Connect RT-PCR Detection System (Bio-Rad, Hercules, CA). The 4-nucleotide insertion caused a significant change in the PCR reaction product melting curve profile, allowing the identification of both homoand heterozygotic mutants. Melting curve analysis was performed using LightCycler 96 SW 1.1 (Roche, Basel, Switzerland) or Precision Melt Analysis (Bio-Rad, Hercules, CA) software. The HRM results were confirmed by sequencing the PCR product, and these samples were used as standards in the subsequent experiments.

\subsection{Drug Treatments}

For drug treatments during the behavioral tests, $2 \times$ concentrated solutions were prepared in E3 medium. The $1.2 \mathrm{mM}$ glutamate (Sigma-Aldrich, catalog no. G1251, Saint Louis, MO, USA) solution was prepared from $30 \mathrm{mM}$ stock. The 3 and $30 \mathrm{mM}$ PTZ (Sigma-Aldrich, catalog no. P6500, Saint Louis, MO, USA) solutions were prepared from $0.5 \mathrm{M}$ stock. These solutions were mixed in a 1:1 proportion with the E3 medium in which the larvae were kept to obtain the final working doses of $600 \mu \mathrm{M}$ glutamate and 1.5 or $15 \mathrm{mM} \mathrm{PTZ}$.

\subsection{Behavioral Experiments}

Before the experiment, the larvae were kept in a Petri dish ( 50 larvae/dish) in an incubator under a $14 \mathrm{~h} / 10 \mathrm{~h}$ light/dark cycle. On the day of the experiment, randomly selected $4 \mathrm{dpf}$ larvae were acclimated to the behavioral testing room for at least $30 \mathrm{~min}$. Locomotor activity was recorded using the ZebraBox high-throughput monitoring system (ViewPoint Life Sciences, Lyon, France). The video files were further analyzed using EthoVision XT software (Noldus, Wageningen, the Netherlands). Data were exported to Microsoft Excel files and further analyzed using Excel (Microsoft, Redmond, WA, USA) and R software (R Foundation for Statistical Computing, Vienna, Austria, R package version 3.6.2). The results for larvae that were not active during the entire recording time (total distance $<10 \mathrm{~mm}$ ) were rejected. Adjusted values of $p<0.05$ were considered statistically significant. The data are expressed as medians with first and third quartiles using boxplots, and dots represent data outliers unless otherwise stated. The numbers of fish that were used in each test are listed in Table 1.

Table 1. Summary of numbers of animals and replications of experiments for all behavioral tests.

\begin{tabular}{|c|c|c|c|c|}
\hline Type of Test & Genotype & Treatment & $\begin{array}{c}\text { Number of } \\
\text { Larvae }\end{array}$ & $\begin{array}{l}\text { Number of } \\
\text { Experiments }\end{array}$ \\
\hline $\begin{array}{l}\text { Open field test adopted for } \\
\text { zebrafish larvae }\end{array}$ & $\begin{array}{c}\mathrm{WT} \\
\operatorname{stim} 2 b^{-/-}\end{array}$ & E3 & $\begin{array}{l}32 \\
33\end{array}$ & 3 \\
\hline Light preference test & $\begin{array}{c}\text { WT } \\
\text { stim } 2 b^{-/-}\end{array}$ & E3 & $\begin{array}{c}10 \\
8\end{array}$ & 5 \\
\hline $\begin{array}{l}\text { Visual-motor response test-no } \\
\text { treatment }\end{array}$ & $\begin{array}{c}\text { WT } \\
\text { stim } 2 b^{-/-}\end{array}$ & E3 & $\begin{array}{l}36 \\
35\end{array}$ & 3 \\
\hline $\begin{array}{l}\text { Visual-motor response } \\
\text { test-glutamate treatment }\end{array}$ & $\begin{array}{c}\text { WT } \\
\text { stim } 2 b^{-/-}\end{array}$ & $600 \mu \mathrm{M}$ glutamate & $\begin{array}{l}36 \\
35\end{array}$ & 3 \\
\hline $\begin{array}{l}\text { Visual-motor response } \\
\text { test-PTZ treatment }\end{array}$ & $\begin{array}{c}\text { WT } \\
\text { stim } 2 b^{-/-} \\
\text {WT } \\
\text { stim } 2 b^{-/}\end{array}$ & $1.5 \mathrm{mM}$ PTZ & $\begin{array}{l}14 \\
18 \\
18 \\
18\end{array}$ & 3 \\
\hline
\end{tabular}

\subsubsection{Open Field Test Adopted for Zebrafish Larvae}

Two minutes before recording locomotor activity, the larvae were transferred to a 12-well plate that was then placed in the ZebraBox. The experiment was performed in a volume of $2 \mathrm{~mL}$ of E3 medium, and the light intensity was set to $70 \%$. Locomotor activity was recorded for $10 \mathrm{~min}$. 
To analyze thigmotaxis, the area was divided into borders and a central area [30]. The experiment was divided into two 5 min periods. The time spent in each part of the well (s), mean total distance traveled $(\mathrm{mm})$, mean velocity $(\mathrm{mm} / \mathrm{s})$, and frequency of fast movements were calculated and compared independently for these two time bins. Paired $t$-tests were used to compare these parameters between the border and central areas within groups. For comparisons between WT and stim $2 b^{-/}$zebrafish, the Wilcoxon rank-sum test was used. Thigmotaxis was based on the time spent in each part of the well and calculated as the following: (duration of movement [in borders or center] + duration of no movement [in borders or center])/(duration of movement [total] + duration of no movement [total]) $\times 100 \%$.

\subsubsection{Light Preference Test}

This experiment was performed as described previously [33]. Two minutes before recording locomotor activity, the larvae were transferred to a Petri dish. Half of the dish was covered with two photographic filters (Cokin P154 ND8, Rungis, France), and the sidewall of this part of the dish was coated with black tape, which together blocked white light from the source above. The chamber was then placed in the ZebraBox. The experiment was performed in a volume of $20 \mathrm{~mL}$ of E3 medium, and the light intensity was set to $70 \%$. Locomotor activity was recorded for $15 \mathrm{~min}$.

The area was divided into dark and light parts. To assess phototaxis, the time spent in each part of the well (s) and mean total distance traveled $(\mathrm{mm})$ in each part were calculated. Paired $t$-tests were used to compare these parameters between the dark and light parts within groups. For comparisons between WT and stim $2 b^{-/-}$zebrafish, the Wilcoxon rank-sum test was used. Phototaxis was assessed based on the time spent in the light part of the dish and calculated as the following: (duration of movement [light part] + duration of no movement [light part])/(duration of movement [total] + duration of no movement [total]) $\times 100 \%$. Depending on this parameter, the responses of zebrafish were divided into three groups: phototaxis ( $>70 \%$ time in light part), no preference ( $>30 \%$ and $<70 \%$ time in light part), and scototaxis $(<30 \%$ time in light part). The distribution of these responses was compared between WT and $\operatorname{sim} 2 b^{-/-}$zebrafish using Pearson's $\chi^{2}$ test.

\subsubsection{Visual-Motor Response Test}

This experiment was performed as described previously [42]. On the day before the experiment, the larvae were placed in 24-well plates that contained $1 \mathrm{~mL}$ of $\mathrm{E} 3$ medium. Thirty minutes before recording locomotor activity, the plates were placed in the ZebraBox. Two minutes before recording, half of the E3 medium volume was exchanged (based on treatment) for E3 medium, glutamate solution, or PTZ solution. The experiment consisted of three phases of the following changes in lighting conditions that were named according to long-lasting changes in zebrafish activity that are induced by these changes: baseline (0-10 $\mathrm{min}, 0 \%$ light intensity), low activity phase (10-20 min, $70 \%$ light intensity), and high activity phase (20-30 min, $0 \%$ light intensity). The mean total distance traveled $(\mathrm{mm})$ and mean velocity $(\mathrm{mm} / \mathrm{s})$ were calculated independently for each of these phases. To compare the low and high activity phases, paired $t$-tests were used. For comparisons between treatments and genotypes, the Wilcoxon rank-sum test was used. For comparisons between more than two groups, the Kruskal-Wallis test was used, followed by the Wilcoxon-Mann-Whitney post hoc test with Benjamini and Hochberg correction. For PTZ treatment, we added to the analysis additional parameters, including the number of seizure-like episodes and amplitude of seizure-like episodes. Seizure-like episodes were defined as events of fast movement at a threshold of $200 \mathrm{~mm} / \mathrm{min}$, accompanied by whirlpool-like circular swimming. The number of these episodes was calculated during the entire duration of the experiment, and the amplitude of these episodes was equal to the distance traveled. The experiments were repeated three times. 


\subsection{In Vivo Imaging of Neuronal Activity}

The in vivo imaging of $\mathrm{Ca}^{2+}$ signals in zebrafish neurons was performed using a Zeiss Lightsheet Z.1 microscope (Zeiss, Oberkochen, Germany, 40× objective, NA $=1.0$ ). Zebrafish that expressed GCaMP5G under the neural promoter $\mathrm{HuC}$ were used in these experiments. Before the experiment, $4 \mathrm{dpf}$ larvae were immobilized using the cholinergic blocker pancuronium bromide $(0.6 \mu \mathrm{g} / \mu \mathrm{L}$; Sigma-Aldrich, catalog no. P1918, Saint Louis, MO, USA) [43] and mounted in 1.5\% low-melting-point agarose (Sigma-Aldrich, catalog no. A9414, Saint Louis, MO, USA). Time lapse images were recorded for $5 \mathrm{~min}$ at $28^{\circ} \mathrm{C}(15 \mathrm{~ms}$ exposure, $1 \mathrm{frame} / \mathrm{s})$. A single plane that contained the habenula, optic tectum, and cerebellum was selected. Oscillations of neuronal $\mathrm{Ca}^{2+}$ signals in the zebrafish larvae brain were observed. Using MATLAB (Mathworks, Natick, MA, USA), changes in fluorescence were extracted from the cells in the periventricular gray zone of the optic tectum (54-57 cells/fish) at single-cell resolution as described elsewhere [44]. Using a function that detects peaks based on changes in slope, peaks of oscillations of $\mathrm{Ca}^{2+}$ levels were selected. Such features as average oscillation frequency and the average amplitude of $\mathrm{Ca}^{2+}$ signals were quantified. The Wilcoxon rank-sum test was applied to compare mutant and WT zebrafish. Adjusted values of $p<0.05$ were considered statistically significant. The data are expressed as medians with first and third quartiles using boxplots, and dots represent data outliers. The following number of cells were analyzed: WT $(n=456)$ and stim $2 b^{-/-}(n=336)$. The following number of animals were analyzed: WT $(n=8)$ and stim $2 b^{-/-}(n=6)$.

\subsection{RNA Sequencing and Real-Time Polymerase Chain Reaction Expression Analysis}

Total RNA was extracted from 5 dpf larvae using TRI Reagent (Invitrogen, catalog no. AM9738, Carlsbad, CA, USA) according to a previously published protocol [45]. Thirty larvae were pooled together to obtain one RNA sample. RNA quality was verified by measuring absorbance at 260, 280 , and $230 \mathrm{~nm}$. Only samples with absorbance $>1.8$ at A260/280 nm and A230/280 nm were used for further processing. Before RNAseq, RNA samples were additionally digested with DNase I and purified using the RNA Clean and Concentrator Kit (ZYMO Research, catalog no. R1013, Irvine, CA, USA) according to the manufacturer's instructions. The sequencing procedure was performed using Illumina methodology. Preparation of the cDNA libraries and sequencing by Next-Generation Sequencing (Illumina, San Diego, CA, USA, NGS NextSeq 500) were performed in cooperation with the Zebrafish Core Facility at the International Institute of Molecular and Cell Biology. The sequencing resulted in approximately $40-70$ million reads per sample with a $76 \mathrm{bp}$ read length. The reads were extracted in FASTQ format and used for the subsequent analysis. The reads were then aligned to zebrafish RefSeq genome assembly annotated genes using the Ensembl annotation. The experiment was performed twice, and two independent samples from WT and stim $2 b^{-/}$larvae were used in each experiment. Gene expression was calculated using the RNAseq by Expectation-Maximization (RSEM) method. Student's $t$-test was used to compare fragments per kilobase per million mapped reads (FPKM)-normalized expression values between the WT and stim $2 b^{-/-}$groups. The $p$ values were corrected for multiple testing. FASTQ files are available in the Sequence Read Archive (accession no. PRJNA627826).

For the RT-PCR analysis of expression, first-strand cDNA was synthesized using the SuperScript IV First-Strand Synthesis System (Invitrogen, catalog no. 18091050, Carlsbad, CA, USA) and 500 ng of RNA templates. RT-PCR was performed in duplicate using $25 \mathrm{ng}$ of cDNA and FastStart Essential DNA Green Master (Roche, catalog no. 06402712001, Basel, Switzerland). The primers that were used for RT-PCR are listed in Table 2. Fold changes were calculated using the ${ }^{\Delta \Delta} \mathrm{Ct}$ method. Eukaryotic translation elongation factor $1 \alpha 1$, like 1 (eef1a1l1) was used as a reference gene. Changes in expression are expressed as fold changes \pm SD using expression in WT as a reference value. The calculations were performed in Microsoft Excel (Microsoft, Redmond, WA, USA). 
Table 2. Primers that were used for Real-Time Polymerase Chain Reaction.

\begin{tabular}{ccc}
\hline Gene & Forward Primer & Reverse Primer \\
\hline eef1a1l1 & AAAATCGGTGGTGCTGGCAA & GGAACGGTGTGATTGAGGGA \\
stim1a & TGAATTCGGATTGCCAGTCGT & TTCAAGTCCCTCTGCGAACC \\
stim $1 b$ & TGAGTTTTGAGGCCATCCGC & AACCCATCCGTCTCTGTCAC \\
stim2a & ATTACGGAGGCGGATCGATT & CCTCAATGCCTCCATCCTGA \\
stim $2 b$ & CTGGTGGAGTGGACGATCTT & CGTCAGAGGAGGTCGAATCA \\
oraila & GTGCATTTTTACCGCTCGCT & TTGAAGAGGCATCTCCCCTC \\
orailb & GCTGTAAGCAACGTGCACAA & TCCCGATGACGGTGGAAAAG \\
orai 2 & CGAGCTAGCCTGGGGTTTTT & AGTCAACCGGCAGGAACTTG \\
\hline
\end{tabular}

\section{Results}

3.1. stim $2 b$ Knockout Does Not Induce a Severe Phenotype in Zebrafish Larvae but Affects Gene Expression and Neuronal Activity

We generated a stim $2 b^{-/-}$mutant zebrafish line using CRISPR/Cas9 technology. The presence of a frameshift mutation was confirmed by gDNA sequencing. The predicted protein was truncated at exon 3 before the transmembrane domain, which resulted in the disruption of interactions with membrane and cytoplasmic targets (Figure 1A,B). The presence of the mutation was confirmed by DNA sequencing and recognized as a shift in the melting curve by HRM analysis (Figure 1C). The development of homozygous stim $2 b^{-/}$zebrafish did not show any irregularities. The viability of larvae between 6 hpf and $5 \mathrm{dpf}$ was very similar to WT fish (Figure 1D). Adults were fertile and survived at least 20 months. As expected, a decrease in stim $2 b$ mRNA levels in the mutant zebrafish was observed. However, no compensatory response by other gene paralogues, such as stim $2 a$, stim1a, or stim1b, occurred, but a slight decrease in stim2a expression was observed (Figure 1E). The downregulation of orai1a and orai2, which encode Oraila and Orai2 channels that are involved in SOCE, was detected.

We next estimated changes in gene expression using RNAseq. A total of 96 genes were downregulated, and 180 were upregulated in stim $2 b^{-/}$larvae compared with WT fish (Table S1). The RNAseq results are available in the Sequence Read Archive (accession no. PRJNA627826). We found several genes that are known to be expressed in the nervous system whose mRNA levels were altered in stim $2 b^{-/}$mutants (Figure 2A, Table 3). Among these genes was annexin3a (anxa3a), which encodes a protein that is known to act as a $\mathrm{Ca}^{2+}$-dependent phospholipid-binding protein. Elevated levels of annexin1c (anxa1c) and annexin5a (anxa5a) were also observed. A few other genes whose products are known to be involved in neural development and function were differentially expressed. These included the upregulation of ECRG4 augurin precursor b (ecrg4b), G protein-coupled receptor 39 (gpr39), and structural maintenance of chromosomes $1 A$ (smc1a) and downregulation of ribonucleotide reductase regulatory subunit M2 (rrm2), neuroguidin (ngdn), period1 a circadian clock (per1a), and homer2. The homer2 gene encodes a protein that is involved in several $\mathrm{Ca}^{2+}$ homeostasis processes in neurons, including the regulation of signaling via metabotropic glutamate receptors.

Next, we analyzed $\mathrm{Ca}^{2+}$ oscillations in neurons in stim $2 b^{+/+}$and stim $2 b^{-/-}$larvae that expressed the GCaMP5G Ca ${ }^{2+}$ probe (Video S1, Video S2). The periventricular gray zone of the optic tectum was chosen as the region of interest. We observed a higher frequency of $\mathrm{Ca}^{2+}$ signal oscillations in stim $2 b^{-/}$larvae (Figure 2B). Moreover, the amplitude of these signals was higher in stim $2 b^{-/}$than in WT zebrafish (Figure 2C). Thus, we investigated whether these changes in expression patterns and neuronal activity correlated with alterations of stim $2 b^{-/-}$zebrafish behavior. 

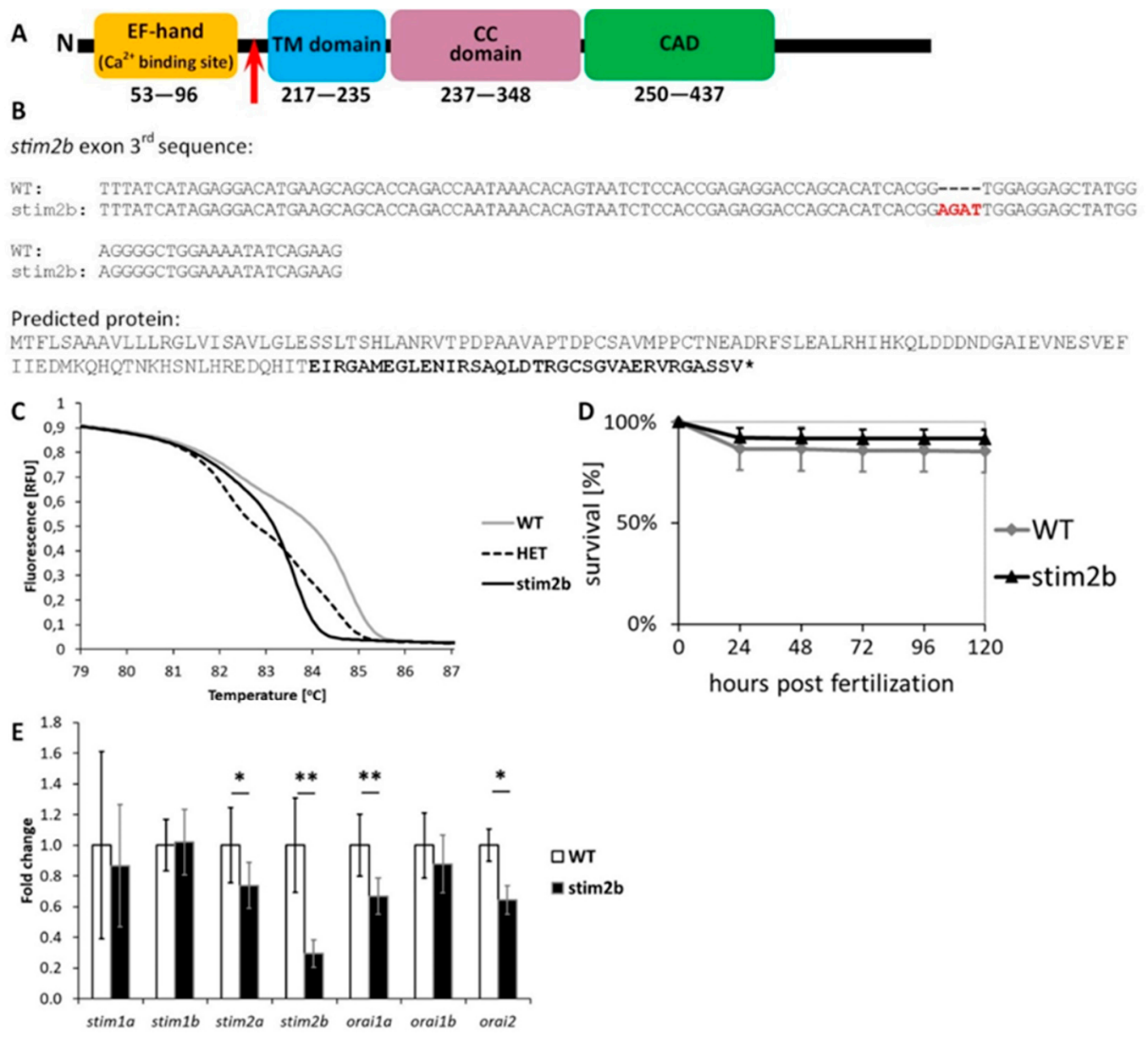

Figure 1. stim $2 b^{-/-}$mutant. (A) Schematic illustration of the most important domains of Stim2 protein. The location of the introduced mutation is marked with a red arrow. (B) DNA sequence of exon 3 of stim $2 b$ in wild-type (WT) and mutant zebrafish (upper) and predicted truncated protein sequence after introducing a frame-shift that cause the mutation (lower). (C) Representative results of the high-resolution melting (HRM) analysis that was used to discriminate mutant (stim2b), heterozygous (HET), and WT individuals. (D) Survival curve that shows the percentage of surviving WT and stim $2 b^{-/}$zebrafish throughout the first 5 days of development. Number of experiments: 3 , survival of 200 larvae of each genotype was estimated during each experiment. (E) RT-PCR results, presented as a mean fold change \pm SD. ${ }^{*} p<0.05,{ }^{* *} p<0.01,{ }^{* * *} p<0.001$, mutant vs. WT (Student's $t$-test). Number of experiments: 8 .

Table 3. RNAseq results of the differential expression of genes that are related to the nervous system in stim $2 b^{-/-}$larvae. Student's $t$-test was used to compare FPKM-normalized expression values between mutant and WT, and the $p$ values were corrected for multiple testing. ${ }^{*} p<0.05$ (FDR $<0.05$ ), ${ }^{* *} p<0.01$ (FDR $<0.05)$, significant difference in fold changes. Number of samples: 4 . Number of experiments: 2.

\begin{tabular}{cccc}
\hline Gene & Mean Fold Change & $\boldsymbol{p}$ & False Discovery Rate \\
\hline anxa1c & 2.13 & 0.0027 & 0.0874 \\
anxa3a & $4.59^{*}$ & 0.0002 & 0.0332 \\
anxa5a & 1.64 & 0.0083 & 0.1387 \\
ecrg4b & $3.65^{*}$ & 0.0001 & 0.0271 \\
gpr39 & $2.87^{*}$ & 0.0003 & 0.0380 \\
homer2 & $0.54^{*}$ & 0.0004 & 0.0426 \\
ngdn & $0.54^{*}$ & 0.0004 & 0.0426 \\
per1a & $0.54^{* *}$ & $<0.0001$ & 0.0066 \\
rrm2 & $0.15^{*}$ & 0.0007 & 0.0500 \\
smc1a & $6.45^{*}$ & $<0.0001$ & 0.0142 \\
\hline
\end{tabular}



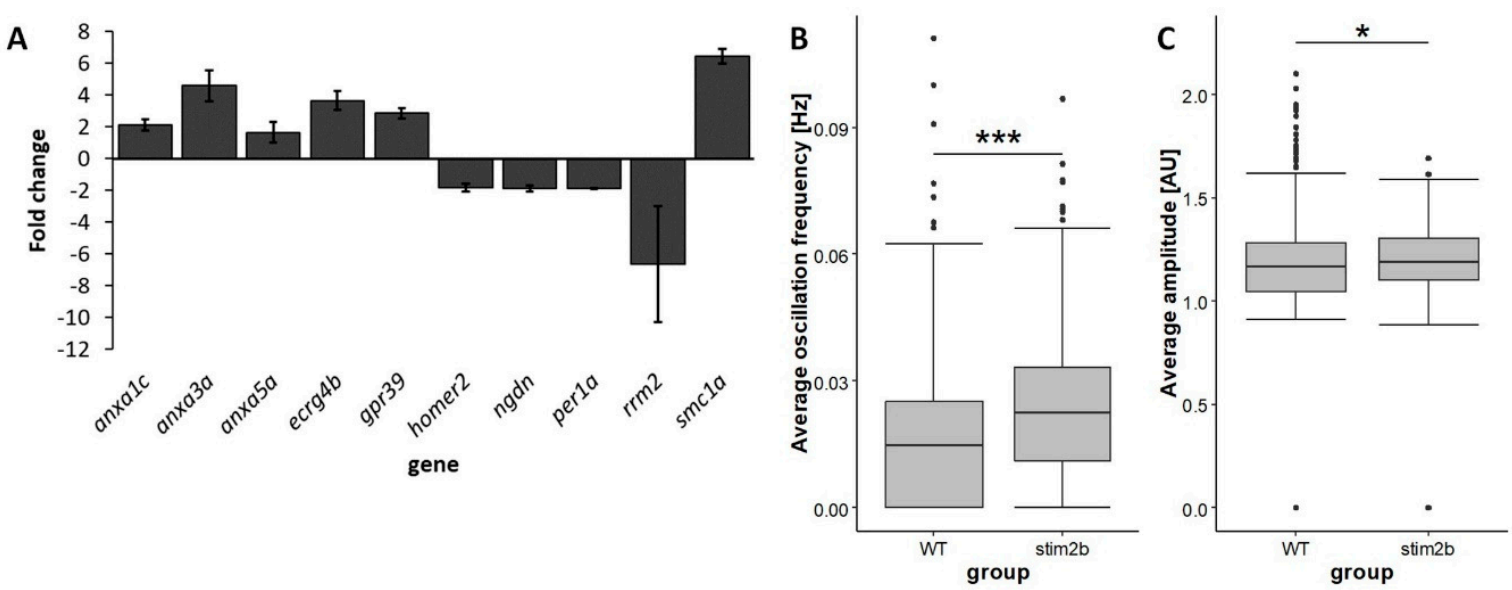

Figure 2. Differential expression of genes that are related to the nervous system and increases in the frequency and amplitude of neuronal $\mathrm{Ca}^{2+}$ oscillations in stim $2 b^{-/-}$larvae. (A) RNAseq results expressed as mean fold changes \pm SD in stim $2 b^{-/-}$samples. The level of expression in WT was used as a reference value $(=1)$. Number of samples: 4 . Number of experiments: 2 . The exact fold change values and statistics are presented in Table 3. (B,C) Box plots of the average frequency $(\mathrm{Hz})(\mathbf{B})$ and average amplitude (AU) (c) of $\mathrm{Ca}^{2+}$ signal oscillations in neurons in the periventricular gray zone of the optic tectum. ${ }^{*} p<0.05,{ }^{* *} p<0.01,{ }^{* * *} p<0.001$, mutant vs. WT (Wilcoxon rank-sum test). Number of cells: 456 (WT) and $336\left(\operatorname{stim} 2 b^{-/-}\right)$. Number of animals: $n=8 \mathrm{WT}, n=6$ stim $2 b^{-/}$.

\section{2. stim2b Knockout Increases Mobility and Thigmotaxis in Zebrafish Larvae}

We first checked basic parameters of locomotor behavior in stim $2 b^{-/-}$larvae. We analyzed zone preference according to a modified method of Schnörr et al. [30] (Figure 3A). This test allows analyses of general characteristics of zebrafish larvae mobility, such as distance traveled and velocity, and thigmotaxis. Zebrafish larvae have a tendency to stay close to borders of the well [31]. This behavior is closely related to anxiety. During the first $5 \mathrm{~min}$, both WT and stim $2 b^{-/-}$fish spent most of the time in proximity to the borders of the well, with similar thigmotactic behavior between groups (Figure 3B, left). Larvae of both genotypes traveled longer distances along the borders of the well (Figure 3C, left) but moved with higher velocity in the central area (Figure 3D, left). After the initial acclimatization period, WT zebrafish began to spend more time in the central area, whereas $s$ tim $2 b^{-/}$zebrafish did not alter their behavior and still moved close to the borders of the well (Figure 3B, right). Mutant larvae also continued to travel a longer distance along the borders of the well and moved with a higher velocity in the central area, in contrast to WT zebrafish (Figure 3C,D, right).

All of the analyzed parameters indicated that $\operatorname{stim} 2 b^{-/}$zebrafish generally exhibited higher mobility than WT zebrafish (Figure 3C-H). Mutant zebrafish covered a longer distance (Figure 3C,D), moved with a higher velocity (Figure $3 \mathrm{E}, \mathrm{F}$ ), and more frequently exhibited high-speed movements (Figure $3 \mathrm{G}, \mathrm{H}$ ) both along the borders and in the central area during both phases of the experiment. 

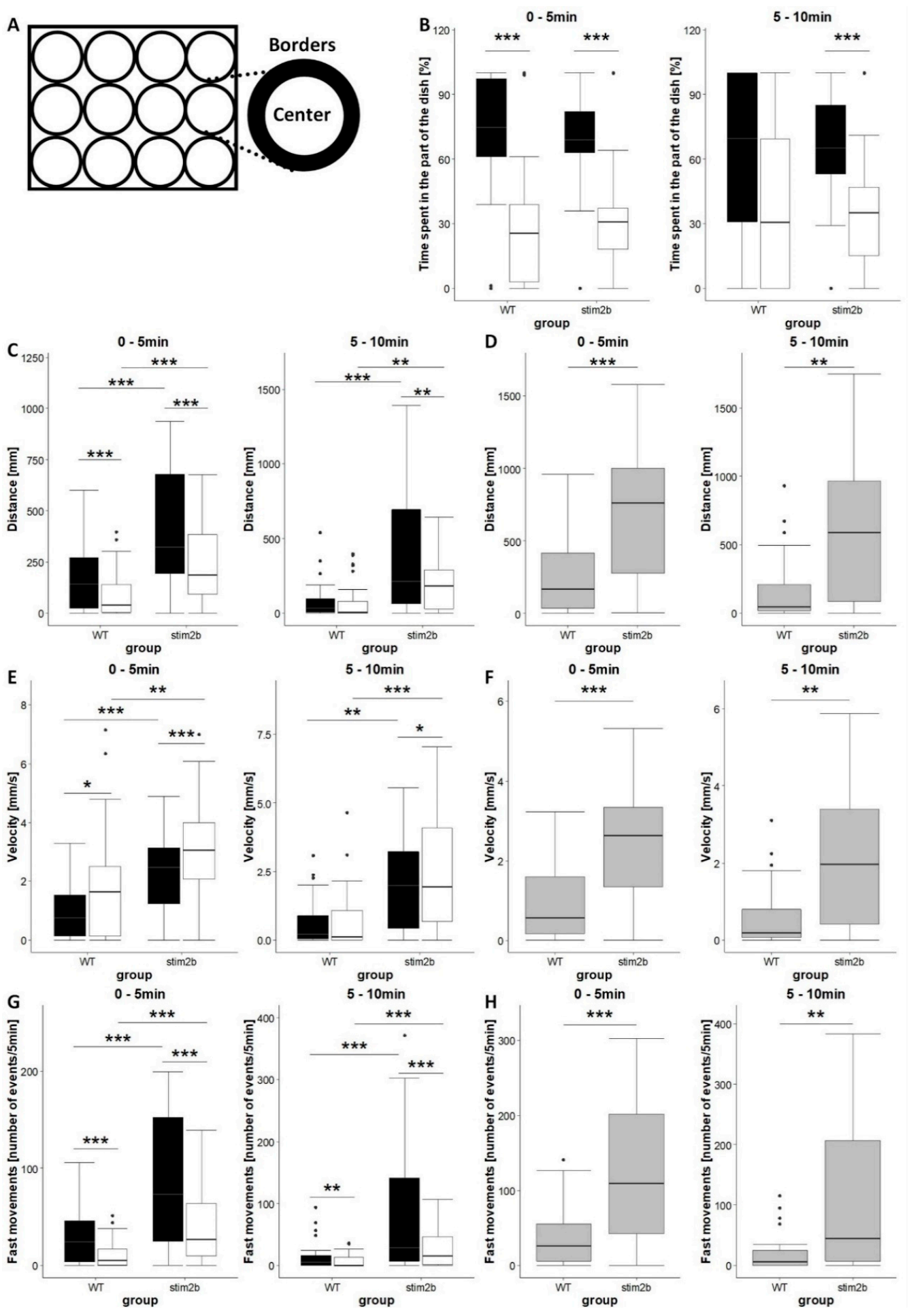

Figure 3. Increases in mobility and thigmotaxis in stim $2 b^{-/-}$larvae in the open field test. (A) Schematic diagram of the 12-well plates that were used in the experiments, showing a division into borders and central areas. (B,C,E,G) Boxplots of the time spent in each area (black in the border; white in center), distance traveled, velocity, and the frequency of fast movements in each part in WT and stim $2 b^{-/-}$ larvae. (D,F,H) Boxplots of distance traveled, velocity, and the frequency of fast movements in WT and $\operatorname{stim} 2 b^{-/}$larvae in both the border and central areas of the well combined. $(\mathbf{G}, \mathbf{H})$ Movements were considered fast movements when velocity was higher than $20 \mathrm{~mm} / \mathrm{s} .{ }^{*} p<0.05,{ }^{* *} p<0.01,{ }^{* * *} p<0.001$, border vs. center for each genotype (paired $t$-test) or mutant vs. WT (Wilcoxon rank-sum test). Number of larvae: $n=32 \mathrm{WT}, n=33$ stim $2 b^{-/-}$. Number of experiments: 3 . 


\section{3. stim $2 b^{-/}$Mutants Exhibited Disruptions of Phototaxis but a Normal Visual-Motor Response}

To further investigate anxiety-like behavior in stim $2 b^{-/}$zebrafish, the light preference test was performed as described previously [32,33] (Figure 4A). Zebrafish larvae exhibited a tendency to stay in the light part and actively avoid the dark part [30]. As expected, WT larvae spent significantly more time and covered a longer distance in the light part than in the dark part of the well (Figure 4B, C). In contrast, stim $2 b^{-/-}$zebrafish exhibited no significant preference for either the light or dark part. The majority of WT larvae (80\%) exhibited clear phototaxis, preferring the light part of the well, and $20 \%$ had no preference (Figure 4 D). Only $60 \%$ of the mutant fish exhibited phototaxis, $15 \%$ had no preference, and $25 \%$ exhibited scototaxis (i.e., preference for dark). The latter behavior was not observed in any WT larvae.
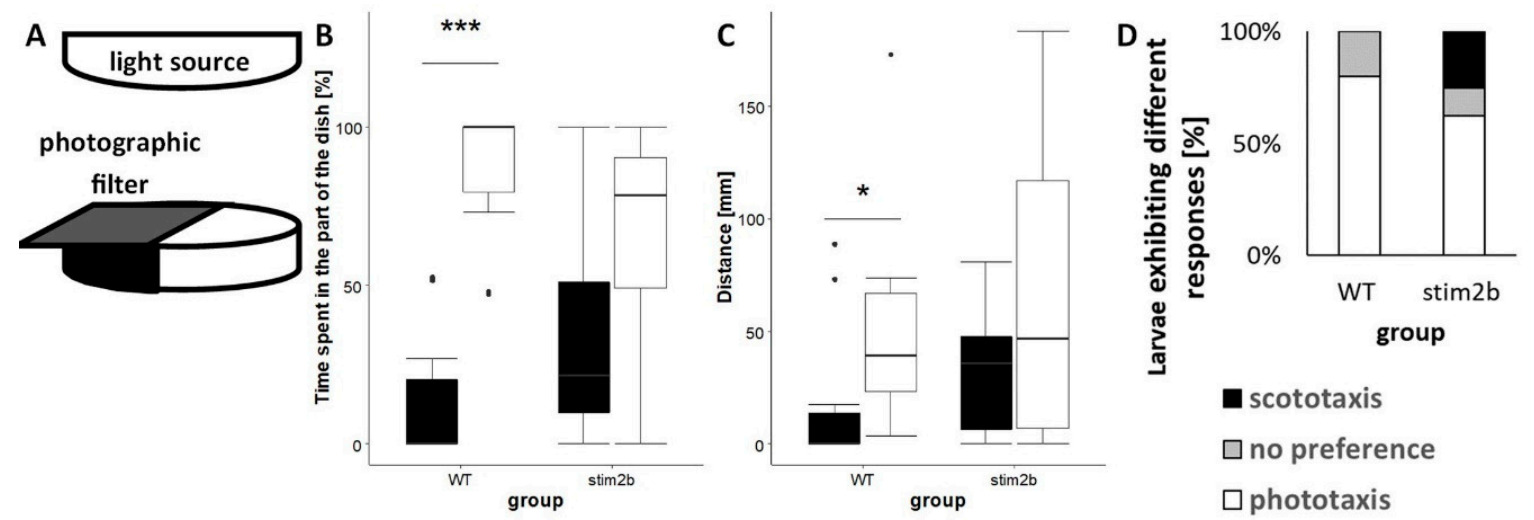

Figure 4. Disruption of phototaxis in $\operatorname{stim} 2 b^{-/-}$larvae in the light preference test. (A) Schematic diagram of the experimental design. The Petri dish was exposed to white light that emanated from above the dish. Illumination of half of the area of the dish was limited using a gray photographic filter. $(\mathbf{B}, \mathbf{C})$ Boxplots of the time spent and distance traveled in each part of the dish (black in the dark part, white in the light part) in WT and stim $2 b^{-/-}$larvae. ${ }^{*} p<0.05,{ }^{* *} p<0.01,{ }^{* * *} p<0.001$, dark vs. light part for each genotype (paired $t$-test). (D) Stacked bar chart of the distribution of different types of responses: phototaxis ( $>70 \%$ time in the light part), no preference $(30-70 \%$ time in the light part), and scototaxis ( $<30 \%$ time in the light part). The distribution of responses was compared using Pearson's $\chi^{2}$ test $(p=0.2419)$. Number of larvae: $n=10 \mathrm{WT}, n=8 \operatorname{stim} 2 b^{-/-}$. Number of experiments: 5 .

To exclude the possibility that disruptions of phototaxis reflected vision deficits, we tested motor responses to sudden changes in light in stim $2 b^{-/-}$larvae using a modified protocol that was described by Liu et al. [42] (Figure 5A). Such a stimulus evokes an immediate startle response in zebrafish larvae, followed by a long-lasting change in activity [46]. As expected, when the light was turned on, WT fish reacted with a startle response, followed by a decrease in mobility (i.e., low activity phase). When the light was turned off, they increased their mobility (i.e., high activity phase; Figure 5B,C). The same response was observed in stim $2 b^{-/}$larvae, indicating that they had a similar ability to differentiate between dark and light as WT fish. Thus, this visual-motor response test excluded the possibility that abnormal phototaxis in stim $2 b^{-/}$mutants resulted from a disruption of light perception. Despite the similar behavior in response to the light stimulus, stim $2 b^{-/-}$larvae exhibited higher activity than WT larvae during all phases of the experiments (Figure 5D,E). These data confirmed that stim $2 b^{-/-}$mutants were hyperactive. 


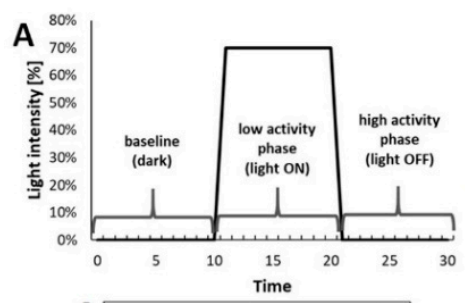

B

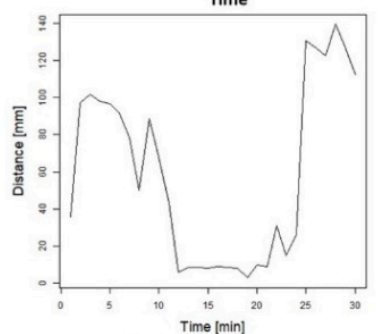

D

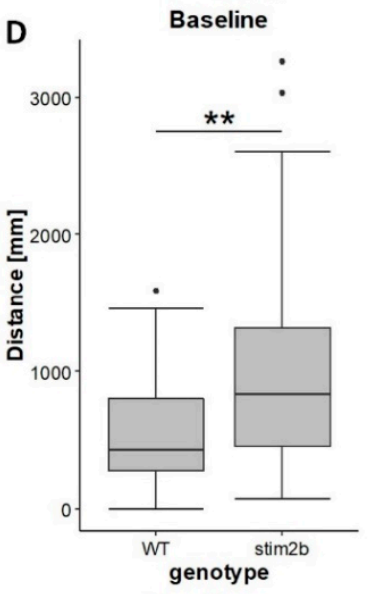

E

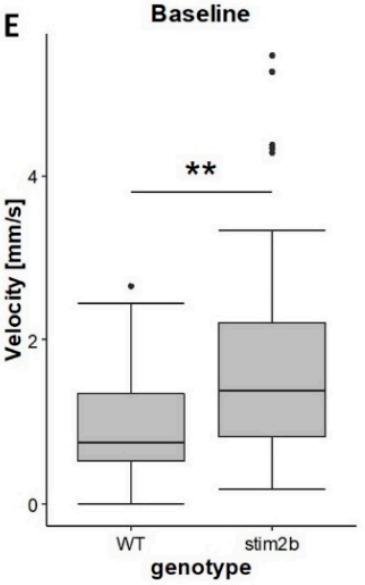

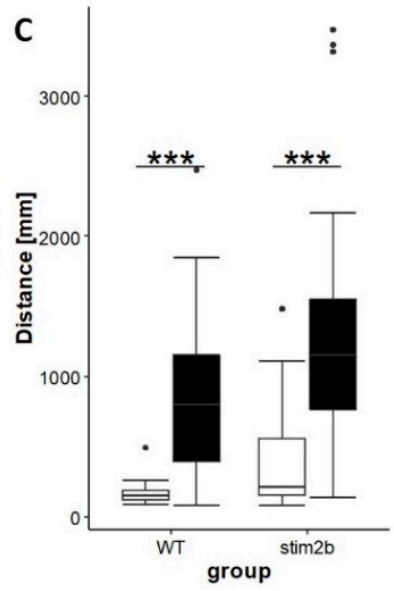

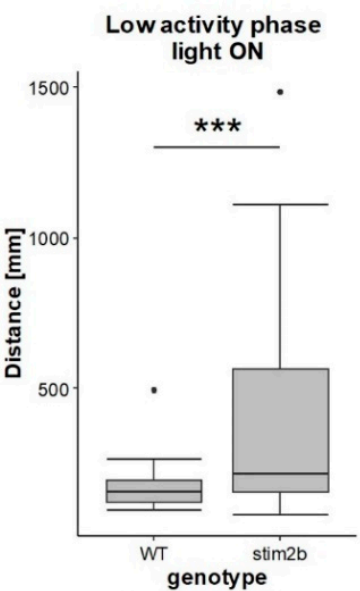

Low activity phase light ON

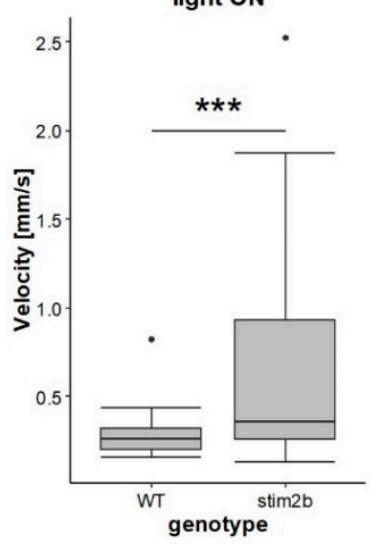

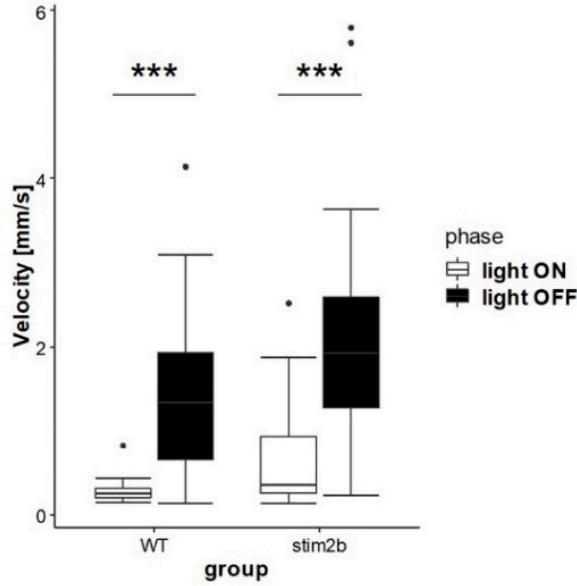

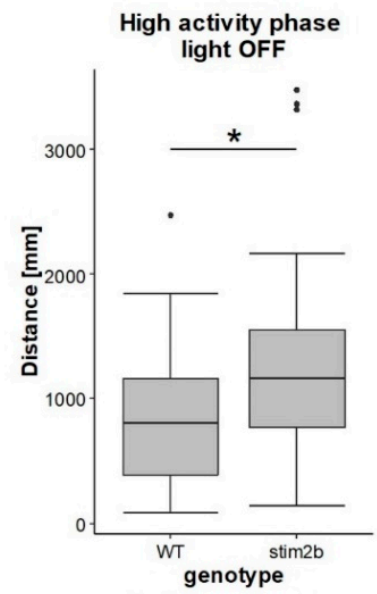

High activ ity phase light OFF

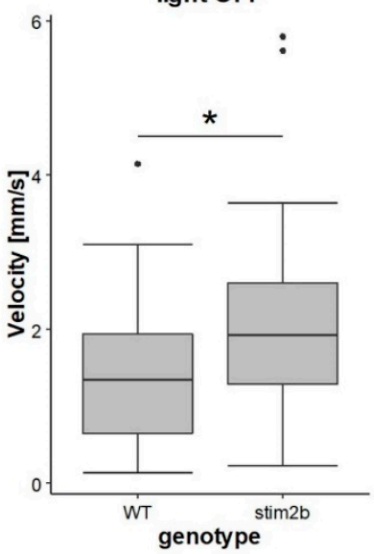

Figure 5. Light perception was unaffected in stim $2 b^{-/}$larvae in the visual-motor response test, indicating hyperactivity of these mutants. (A) Experimental design that shows changes in light intensity during the experiment. Larva activity was recorded during a $30 \mathrm{~min}$ period that consisted of a baseline phase ( $0 \%$ light), inactive phase ( $70 \%$ light), and high activity phase $(0 \%$ light). (B) Representative plot of the distance traveled by a single larva during $1 \mathrm{~min}$ of the experiment. The plot shows the response of WT larvae. (C) Boxplots of the distance traveled and velocity in WT and stim $2 b^{-/-}$larvae after turning the light on (in white) and off (in black). ${ }^{*} p<0.05,{ }^{* *} p<0.01,{ }^{* * *} p<0.001$, response to turning the light on vs. off for each genotype (paired $t$-test). (D) Boxplots of the distance traveled and (E) velocity in WT and stim $2 b^{-/-}$larvae during each phase of the experiment. ${ }^{*} p<0.05,{ }^{* *} p<0.01$, *** $p<0.001$, mutant vs. WT (Wilcoxon rank-sum test). Number of larvae: $n=36 \mathrm{WT}, n=35 \mathrm{stim}^{-b^{-/}}$. Number of experiments: 3 . 


\subsection{Response to GABAergic and Glutamatergic Signaling Modulators is Affected by stim2b Knockout}

The increase in mobility, the presence of circling behavior, and neuronal hyperactivity in stim $2 b^{-/}$ mutants suggested the presence of seizure-like activity. We next investigated the response of stim $2 b^{-/-}$larvae to PTZ, a drug that is used to induce seizures. We expected that blocking inhibitory $\gamma$-aminobutyric acid (GABA)ergic signaling with PTZ would reveal greater susceptibility to seizures in mutant fish. Using the visual-motor response test (described in Section 3.3), we first tested different doses of PTZ. We found that $1.5 \mathrm{mM}$ PTZ had a weak effect, whereas $15 \mathrm{mM}$ PTZ significantly increased activity and induced the presence of rapid "whirlpool-like" circular swimming behavior in both WT and stim $2 b^{-/-}$larvae (Figure 6). Upon stimulation with the lower dose of PTZ, WT larvae exhibited an increase in mobility only during the first $10 \mathrm{~min}$ of the test, whereas stim $2 b^{-/-}$larvae traveled a longer distance and had a higher velocity also during high activity phase (Figure 6A,B). The response to treatment with the high dose of PTZ was lower in stim $2 b^{-/}$mutants than in WT larvae. Both WT and stim $2 b^{-/}$larvae, increased their mobility upon $15 \mathrm{mM}$ PTZ treatment, however during the baseline and low activity phases caused $\operatorname{stim} 2 b^{-/-}$larvae to move a shorter distance and have a lower velocity than WT larvae (Figure 6A,B). We analyzed the occurrence of seizure-like episodes, which were characterized by a high velocity and long distance traveled. The mean amplitude of seizure-like episodes, defined as the distance traveled, was higher in WT fish (Figure 6C). These results showed that stim $2 b^{-/-}$larvae exhibited greater susceptibility to seizures with a low dose of PTZ, but they exhibited a weaker response to the high dose of PTZ.

We next investigated the response of mutant and WT zebrafish to glutamate. Glutamate signaling plays an important role in epileptogenesis [47], and STIM proteins were shown to affect glutamatergic transmission $[19,48,49]$. We used the same protocol as for PTZ treatment to investigate the effect of glutamate on mobility in the low and high activity phases. Both WT and mutant zebrafish that were treated with $600 \mu \mathrm{M}$ glutamate exhibited a significant increase in activity, reflected by a longer distance traveled and higher velocity (Figure 7). When the light was turned on (i.e., low activity phase), $\operatorname{stim} 2 b^{-/-}$mutants that were treated with glutamate traveled a longer distance and had a higher velocity compared with WT zebrafish. When the light was turned off (i.e., high activity phase), the distance traveled and velocity in stim $2 b^{-/-}$larvae that were treated with glutamate did not differ from WT larvae. In summary, a stronger response to glutamate was observed in stim $2 b^{-/-}$mutants only under conditions of sudden light exposure immediately after the startle response. This suggests that higher activity in stim $2 \mathrm{~b}^{-/-}$mutants might be attributable to the dysregulation of neuronal $\mathrm{Ca}^{2+}$ homeostasis. 

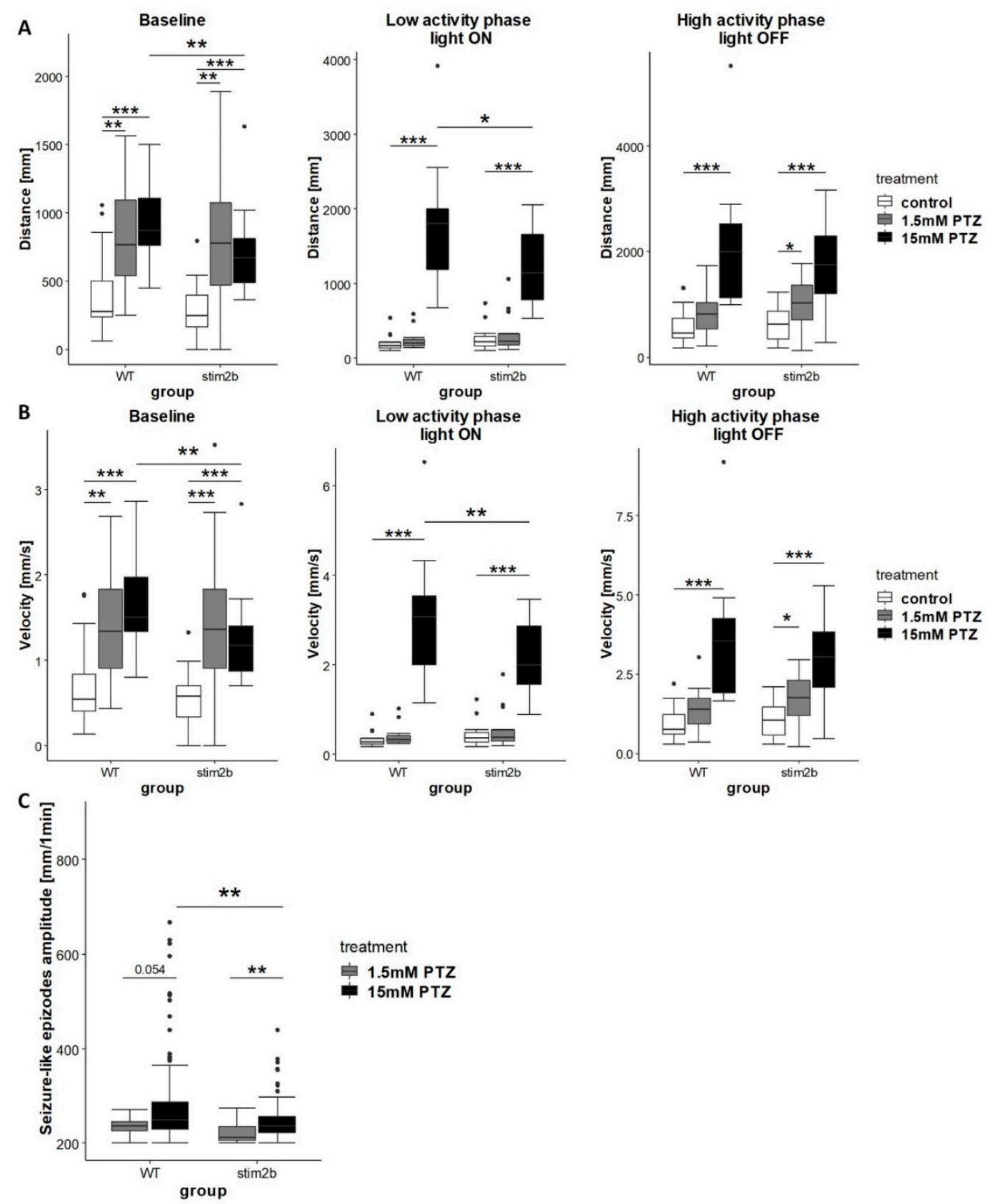

Figure 6. Treatment with the low dose of pentylenetetrazol (PTZ) induced stronger reaction of stim $2 b^{-/-}$ than WT larvae and treatment with high dose of PTZ induce weaker reaction of stim $2 b^{-/-}$than WT larvae. The experimental design was the same as described in Figure 5A. Larva activity was recorded during a $30 \mathrm{~min}$ period that consisted of a baseline phase ( $0 \%$ light), low activity phase (70\% light), and high activity phase ( $0 \%$ light). Before the experiment, half of the medium in the dish was exchanged with PTZ solution (final concentration: 1.5 or $15 \mathrm{mM}$ ). (A,B) Boxplots of the distance traveled (A) and velocity (B) in WT and stim $2 b^{-/-}$larvae during all phases of the experiment. (C) Boxplots of the average amplitude (distance traveled during $1 \mathrm{~min}$ ) of seizure-like episodes that occurred during the experiment in WT and stim $2 b^{-/-}$larvae. Seizure-like episodes were defined as events of a long distance traveled within a short period of time ( $>200 \mathrm{~mm}$ within $1 \mathrm{~min}) .{ }^{*} p<0.05,{ }^{* *} p<0.01,{ }^{* * *} p<0.001$, PTZ-treated larvae vs. control group of the same genotype (Wilcoxon-Mann-Whitney post hoc test with Benjamini and Hochberg correction) or mutant vs. WT with the same treatment (Wilcoxon rank-sum test). Number of larvae: $n=18$ for all groups, with the exception of $n=14 \mathrm{WT}$ that were treated with 1.5 mM PTZ. Number of experiments: 3. 

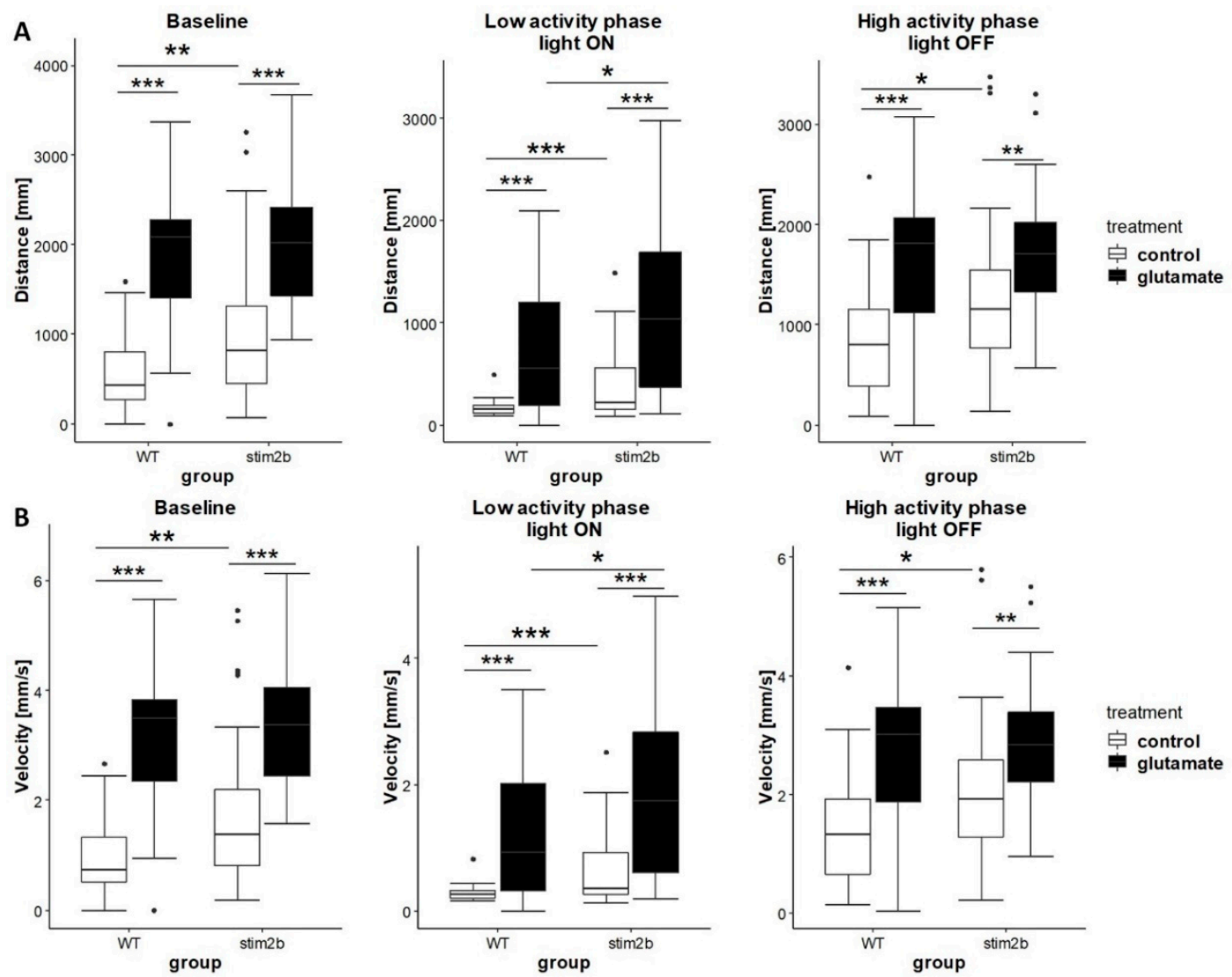

Figure 7. Glutamate treatment induced a stronger response in stim $2 b^{-/}$mutants than in WT larvae. The experimental design was the same as described in Figure 5A. Activity was recorded during a $30 \mathrm{~min}$ period that consisted of a baseline phase ( $0 \%$ light), low activity phase ( $70 \%$ light), and high activity phase $(0 \%$ light). Before the experiment, half of the medium in the dish was exchanged with a glutamate solution (final concentration: $600 \mu \mathrm{M})$. (A,B) Boxplots of the distance traveled (A) and velocity (B) in WT and stim $2 b^{-/-}$larvae during all phases of the experiment. ${ }^{*} p<0.05,{ }^{* *} p<0.01$, *** $p<0.001$, glutamate-treated larvae vs. control group of the same genotype or mutant vs. WT (Wilcoxon rank-sum test). Number of larvae: $n=36 \mathrm{WT}, n=35$ stim $2 b^{-/}$. Number of experiments: 3 .

\section{Discussion}

STIM2 protein is essential for SOCE in hippocampal neurons [17]. In cortical neurons that were isolated from Stim2 $2^{-/-}$mice, basal $\mathrm{Ca}^{2+}$ levels and SOCE decreased [25]. However, the role of STIM2 in shaping behavior remains unclear. Early lethality in mice that lack STIM2 $[25,26]$ makes such studies challenging. To address this problem, we took advantage of zebrafish genetics, which possess two genes that encode Stim2 (stim $2 a$ and stim $2 b$ ). We created a zebrafish line with stim $2 b$ knockout, which appeared to be viable and fertile while having only a weak neurological phenotype. In these mutant zebrafish, we detected the significant downregulation of stim $2 a$, oraila, and orai2 and non-sense-mediated decay of the stim $2 b$ transcript. Thus, we cannot exclude the possibility that the observed phenotypes (e.g., increase in thigmotaxis, disruption of phototaxis, differential responses to PTZ and glutamate, a higher frequency of $\mathrm{Ca}^{2+}$ spikes, and a higher amplitude of $\mathrm{Ca}^{2+}$ spikes) resulted from the dysregulation of SOCE rather than a deficiency of Stim2b itself. However, the presence of functional Stim2a in the mutant fish might obscure the effect of stim $2 b$ knockout. In addition to changes in the levels of mRNA of SOCE components, we found the differential expression of several genes that encode proteins that are important for the function and development of the nervous system. The differential expression of these genes might be responsible for the observed behavioral changes in mutant zebrafish. 
One of the upregulated genes was anxa3a, the levels of which were 4.5-times higher in mutant larvae than in WT larvae. Additionally, anxa1c and anxa5a expression was slightly higher in stim $2 b^{-/}$ larvae. Notably, annexins upregulation was observed in epileptic brains [50,51]. A significant increase in ANXA3 levels was also found in brain injuries [52,53]. Expression of the ecrg $4 b$ gene, which encodes Augurin protein, was also elevated. Augurin is involved in nervous system development [54] and was shown to be upregulated in brain samples that were isolated from aged mice [55] and mice that overexpressed Tau [56]. Another gene that was upregulated in mutant larvae was gpr39. This gene belongs to the ghrelin receptor family and encodes a G-protein-coupled receptor that senses changes in extracellular zinc ion levels. Signaling via GPR39 may reduce excitatory activity during seizure-related excessive neuronal firing that is caused by enhanced GABAergic responses [57]. The higher level of gpr39 expression that was observed in the present study may affect inhibitory signaling in stim $2 b^{-/}$ larvae and protect against excessive neuronal activity that is induced by high-dose PTZ stimulation. GPR39 was also shown to modulate anxiety-like behavior, and GPR39 expression was increased by antidepressant administration [58]. A very high six-fold increase in smc1a expression was observed, which encodes a member of the cohesion complex that is responsible for chromosome segregation during cell division. The downregulation of smc1a impaired neuronal development in zebrafish [59], and mutations of this gene were found in patients with epilepsy $[60,61]$.

Four genes that were downregulated are potentially interesting: $r r m 2, n g d n$, per1a, and homer2. Rrm2 is involved in DNA replication and was shown to be crucial for proper development of the nervous system in zebrafish. A mutation of $r r m 2$ resulted in the disorganization of forebrain glia and severe axonal pathfinding errors in zebrafish larvae [62]. The $n g d n$ gene encodes a translational regulatory protein that is active during development of the vertebrate nervous system and plays a role in synaptic plasticity [63]. The per1a gene is involved in photoperiodism. Interestingly, the downregulation of its paralogue, per1b, resulted in an increase in motor activity in zebrafish larvae and aberrant development of the dopaminergic system [64]. The homer2 gene encodes a protein that is involved in synaptic plasticity and regulates neurotransmission [65]. It is involved in $\mathrm{Ca}^{2+}$ homeostasis by regulating the activity of proteins that are involved in $\mathrm{Ca}^{2+}$ signaling, including metabotropic glutamate receptors, inositol triphosphate receptors, and transient receptor potential channels [66,67]. It also participates in regulating basal cytosolic $\mathrm{Ca}^{2+}$ via an interaction with the plasma membrane calcium reuptake pump [68]. The decrease in Homer2 levels appeared to be responsible for the observed changes in the response to glutamate stimulation in stim $2 b^{-/-}$larvae.

Only a few studies have investigated the role of STIM2 in behavior, but the results have been inconclusive. Mice that overexpressed STIM2 and ORAI1 in neurons exhibited reductions of anxiety-like behavior, including increases in exploration of the arena in the open field test and time spent on the open arms of the elevated plus maze [69]. Mice with double Stim1/Stim2 conditional knockout in the forebrain spent more time on the open arms of the elevated plus maze, suggesting a greater propensity to engage in exploration and a decrease in anxiety-like behavior [27], thus Stim1/Stim2 knockout in mice gave a similar effect as STIM2 and ORAI1 overexpression. In the present study, zebrafish larvae that were devoid of Stim $2 b$ exhibited an increase in mobility after they were transferred to a new dish, and thigmotactic behavior lasted longer in mutant larvae than in WT larvae. Thigmotaxis is considered to be related to anxiety [28,31]. Therefore, the results of the thigmotaxis test indicated an increase in anxiety-like behavior in stim $2 b^{-/}$zebrafish. To investigate whether stim $2 b^{-/-}$mutants exhibited an anxiety-related phenotype, we performed the light preference test. The tendency of zebrafish larvae to avoid darkness is positively correlated with their anxiety level [28]. This test showed the disruption of phototaxis in stim $2 b^{-/}$zebrafish and also an opposite tendency (i.e., scototaxis) in some fish. Thus, contrary to the results of the open field test, this observation suggested a decrease in anxiety-like behavior. The visual-motor response test was performed [42] to exclude the possibility that $\operatorname{sim} 2 \mathrm{~b}^{-/-}$ mutants had difficulties in visually distinguishing between dark and light. The results indicated that the disrupted phototaxis in stim $2 b^{-/}$larvae was not attributable to visual impairments. Moreover, the visual-motor response test showed that $\operatorname{sim} 2 b^{-/-}$larvae were hyperactive during all phases of the 
test, independent of the lighting conditions. Similar hyperactivity was observed in zebrafish larvae in models of attention-deficit/hyperactivity disorder (ADHD), such as micall2b knockout [70] and lphn3.1 knockdown [71]. Notably, variations of STIM2 copy number were observed in ADHD patients [72], and deficits in learning ability that are observed in Stim $2^{-1-}$ mice [25] are also a feature of ADHD.

An increase in thigmotaxis might be related not only to an increase in anxiety, but it also can be an indicator of circling behavior. In a previous study, PTZ-treated larvae exhibited an increase in mobility and swimming abnormalities, characterized by circular trajectories [35]. Disruptions of phototaxis may also be a sign of circling behavior in stim $2 b^{-/-}$larvae, which does not depend on light. Thus, the changes in behavior that were observed in $\operatorname{stim} 2 b^{-/-}$mutants in the present study may be related to seizure-like activity. A similar phenotype (i.e., increase in thigmotaxis and decrease in phototaxis) was also previously observed in tsc mutant larvae, which had greater susceptibility to PTZ-induced epileptogenesis [33]. Larvae with depdc5 knockdown exhibited hyperactivity early in development (28 hpf) and aberrant locomotion with an increase in circular swimming ( $3 \mathrm{dpf})$ that was correlated with neuronal hyperactivity [73].

We also found that $\operatorname{stim} 2 b^{-/-}$larvae exhibited a greater frequency and amplitude of $\mathrm{Ca}^{2+}$ oscillations in the periventricular zone of the optic tectum. This structure is involved in processing visual stimuli and the activation of motor responses. Therefore, disruption of the phototactic response may be attributable to an increase in activity in this region. Disruption of the phototactic response in $\operatorname{sim} 2 \mathrm{~b}^{-/-}$ mutant zebrafish and their increase in mobility may have occurred because their neuronal network did not properly develop. This defect may result from the dysregulation of genes that are important for neurodevelopment, such as $r r m 2$, $n g d n$, and smc1a. Notably, an increase in neuronal activity upon seizure induction was observed in the optic tectum in zebrafish [74]. Therefore, abnormal behavior in $\operatorname{stim} 2 b^{-/}$larvae, combined with neuronal hyperactivity and the upregulation of annexin gene expression, suggested seizure-like activity in these mutants.

Greater susceptibility to PTZ treatment is observed in genetic zebrafish models of epilepsy. Therefore, we investigated the response of $\operatorname{sim} 2 b^{-/-}$larvae to this drug. We found that $\operatorname{sim} 2 b^{-/-}$larvae responded to the low dose of PTZ with a higher distance traveled and velocity in the high activity phase of the visual-motor response test. In contrast, stim $2 b^{-/-}$larvae that were treated with the high dose of PTZ reacted with a shorter distance traveled and velocity compared with WT zebrafish in the low activity phase of the test. These results indicated the distinct sensitivity of mutant larvae to PTZ compared with WT zebrafish, thus further indicating that the mutants exhibited seizure-like activity.

To further unveil the possible mechanism of behavioral alterations in stim $2 b^{-/-}$larvae, we observed their response to glutamate stimulation. On the basis of the available literature we could suggest that the glutamate is able to reach neurons in zebrafish larvae [75]. However, the effect of glutamate treatment can be a result of stimulation taste receptors. Upon glutamate treatment, we observed an increase in mobility in both WT and mutant zebrafish, but stim $2 b^{-/-}$mutants were more active during the inactive phase of the visual-motor response test. Changes in gpr39 and homer2 levels can affect neuronal excitability and may contribute to hyperactivity of $\operatorname{stim} 2 b^{-/-}$larvae and greater sensitivity to PTZ and glutamate treatment. STIM2 has been shown to have complex actions on glutamate signaling. STIM2 was shown to increase glutamate signaling via $\alpha$-amino-3-hydroxy-5-methyl-4-isoxazolepropionic acid receptors $[19,48]$. The downregulation of STIM2 enhanced $\mathrm{Ca}^{2+}$ influx via NMDA receptors [49]. Therefore, the higher responsiveness of $\operatorname{sim} 2 b^{-/-}$zebrafish may be related to an increase in NMDA receptor activity. However, the effects of STIM2 on voltage-gated calcium channels cannot be excluded, although no such data are available. STIM1 was shown to affect the activity of voltage-gated calcium channels through interactions with the SOAR domain that shares $\sim 82 \%$ sequence identity with STIM2 and $84 \%$ sequence identity with the zebrafish Stim2b SOAR (according to Ensembl release 99 [36]).

\section{Conclusions}

We created $\operatorname{stim} 2 b^{-/}$zebrafish that did not present a severe phenotype or lower viability. We speculate that the dysregulation of SOCE affects gene expression. Such effects have been previously 
described (reviewed in [9]). We observed the differential expression of several genes in stim $2 b^{-1}$ larvae. Among these genes were $r m m 2, n g d n$, and $s m c 1 a$, whose aberrant levels may affect the development of brain structures in stim $2 b^{-/-}$larvae. Moreover, neuronal activity in these mutants was affected, with a higher frequency and amplitude of $\mathrm{Ca}^{2+}$ oscillations than in WT larvae. The behavioral experiments indicated an increase in mobility and the occurrence of circling behavior in stim $2 b^{-/}$zebrafish. Our findings suggest that stim $2 b^{-1-}$ larvae exhibited seizure-like activity, which was confirmed by their stronger responses to treatment with glutamate and a low dose of PTZ. However, the mechanism that links Stim $2 \mathrm{~b}$ with the higher frequency of neuronal $\mathrm{Ca}^{2+}$ oscillations and behavioral changes (e.g., hyperactivity and seizure susceptibility) is unclear and requires further investigation.

Supplementary Materials: The following are available online at http://www.mdpi.com/2073-4409/9/5/1285/s1, Table S1: RNAseq results of the differential expression of genes in stim $2 b^{-/-}$larvae. Video S1: $\mathrm{Ca}^{2+}$ oscillations in neurons of stim $2 b^{+/+}$. Video S2: $\mathrm{Ca}^{2+}$ oscillations in neurons of $\operatorname{stim} 2 b^{-/}$.

Author Contributions: Conceptualization, I.W. and J.K.; methodology, I.W., R.K.G., B.W. and J.K.; software, I.W., R.K.G. and B.W.; formal analysis, I.W., R.K.G. and B.W.; investigation, I.W., R.K.G., O.P. and J.K.; resources, J.K.; data curation, I.W. and R.K.G.; writing-original draft preparation, I.W. and J.K.; writing-review and editing, I.W., R.K.G., B.W., O.P. and J.K.; visualization, I.W. and R.K.G; supervision, J.K.; project administration, J.K.; funding acquisition, J.K. All authors read and agreed to the published version of the manuscript.

Funding: This research and APC was funded by the National Science Centre (no. 2016/23/B/NZ3/03142).

Acknowledgments: The project (OPUS12 to JK) was financed by the National Science Centre (no. 2016/23/B/NZ3/03142). We thank Vladimir Korzh and Tomasz Wẹgierski for critically reading the manuscript. We thank Dominika Dubicka-Boroch for administrative support, Łukasz Majewski for managing laboratory operations, Katarzyna Misztal for running the sequencing experiments, and the personnel at the Zebrafish Core Facility at the International Institute of Molecular and Cell Biology (Warsaw, Poland) for maintenance of the fish lines. I.W. and R.K.G. are students at the Postgraduate School of Molecular Medicine, Warsaw Medical University.

Conflicts of Interest: The authors declare no conflict of interest.

\section{References}

1. Williams, R.T.; Manji, S.S.M.; Parker, N.J.; Hancock, M.S.; Van Stekelenburg, L.; Eid, J.-P.; Senior, P.V.; Kazenwadel, J.; Shandala, T.; Saint, R.; et al. Identification and characterization of the STIM (stromal interaction molecule) gene family: Coding for a novel class of transmembrane proteins. Biochem. J. 2001, 357, 673. [CrossRef] [PubMed]

2. Erro, A.B.; Jardin, I.; Salido, G.M.; Rosado, J.A. Role of STIM2 in cell function and physiopathology. J. Physiol. 2017, 595, 3111-3128. [CrossRef] [PubMed]

3. Cai, X. Molecular Evolution and Functional Divergence of the $\mathrm{Ca}^{2+}$ Sensor Protein in Store-operated $\mathrm{Ca}^{2+}$ Entry: Stromal Interaction Molecule. PLoS ONE 2007, 2, e609. [CrossRef] [PubMed]

4. Wasilewska, I.; Gupta, R.K.; Palchevska, O.; Kuznicki, J. Identification of Zebrafish Calcium Toolkit Genes and their Expression in the Brain. Genes 2019, 10, 230. [CrossRef]

5. Skibinska-Kijek, A.; Wisniewska, M.B.; Gruszczynska-Biegala, J.; Methner, A.; Kuznicki,J. Immunolocalization of STIM1 in the mouse brain. Acta Neurobiol. Exp. 2009, 69, 413-428.

6. Steinbeck, J.A.; Henke, N.; Opatz, J.; Gruszczynska-Biegala, J.; Schneider, L.; Theiss, S.; Hamacher, N.; Steinfarz, B.; Golz, S.; Brüstle, O.; et al. Store-operated calcium entry modulates neuronal network activity in a model of chronic epilepsy. Exp. Neurol. 2011, 232, 185-194. [CrossRef]

7. Tu, C.-C.; Wan, B.-Y.; Zeng, Y. STIM2 knockdown protects against ischemia/reperfusion injury through reducing mitochondrial calcium overload and preserving mitochondrial function. Life Sci. 2020, 247, 116560. [CrossRef]

8. Brandman, O.; Liou, J.; Park, W.S.; Meyer, T. STIM2 Is a Feedback Regulator that Stabilizes Basal Cytosolic and Endoplasmic Reticulum Ca ${ }^{2+}$ Levels. Cell 2007, 131, 1327-1339. [CrossRef]

9. Majewski, L.; Kuznicki, J. SOCE in neurons: Signaling or just refilling? Biochim. Biophys. Acta 2015, 1853, 1940-1952. [CrossRef]

10. Wegierski, T.; Kuznicki, J. Neuronal calcium signaling via store-operated channels in health and disease. Cell Calcium 2018, 74, 102-111. [CrossRef]

11. Berridge, M.J. Neuronal Calcium Signaling. Neuron 1998, 21, 13-26. [CrossRef] 
12. Moccia, F.; Zuccolo, E.; Soda, T.; Tanzi, F.; Guerra, G.; Mapelli, L.; Lodola, F.; D'Angelo, E. Stim and Orai proteins in neuronal $\mathrm{Ca}^{2+}$ signaling and excitability. Front. Cell. Neurosci. 2015, 9, 153. [CrossRef] [PubMed]

13. Gruszczynska-Biegala, J.; Pomorski, P.; Wisniewska, M.B.; Kuznicki, J. Differential Roles for STIM1 and STIM2 in Store-Operated Calcium Entry in Rat Neurons. PLoS ONE 2011, 6, e19285. [CrossRef] [PubMed]

14. Gruszczynska-Biegala, J.; Kuznicki, J. Native STIM2 and ORAI1 proteins form a calcium-sensitive and thapsigargin-insensitive complex in cortical neurons. J. Neurochem. 2013, 126, 727-738. [CrossRef] [PubMed]

15. Baba, A.; Yasui, T.; Fujisawa, S.; Yamada, R.X.; Yamada, M.; Nishiyama, N.; Matsuki, N.; Ikegaya, Y. Activity-Evoked Capacitative $\mathrm{Ca}^{2+}$ Entry: Implications in Synaptic Plasticity. J. Neurosci. 2003, 23, 7737-7741. [CrossRef] [PubMed]

16. LaLonde, J.; Saia, G.; Gill, G. Store-Operated Calcium Entry Promotes the Degradation of the Transcription Factor Sp4 in Resting Neurons. Sci. Signal. 2014, 7, ra51. [CrossRef]

17. Sun, S.; Zhang, H.; Liu, J.; Popugaeva, E.; Xu, N.-J.; Feske, S.; White, C.L.; Bezprozvanny, I.B. Reduced synaptic STIM2 expression and impaired store-operated calcium entry cause destabilization of mature spines in mutant presenilin mice. Neuron 2014, 82, 79-93. [CrossRef]

18. Park, C.Y.; Shcheglovitov, A.; Dolmetsch, R. The CRAC Channel Activator STIM1 Binds and Inhibits L-Type Voltage-Gated Calcium Channels. Science 2010, 330, 101-105. [CrossRef]

19. Garcia-Alvarez, G.; Lu, B.; Yap, K.A.F.; Wong, L.C.; Thevathasan, J.V.; Lim, L.; Ji, F.; Tan, K.W.; Mancuso, J.J.; Tang, W.; et al. STIM2 regulates PKA-dependent phosphorylation and trafficking of AMPARs. Mol. Biol. Cell 2015, 26, 1141-1159. [CrossRef]

20. Gemes, G.; Bangaru, M.L.Y.; Wu, H.-E.; Tang, Q.; Weihrauch, R.; Koopmeiners, A.S.; Cruikshank, J.M.; Kwok, W.-M.; Hogan, Q.H. Store-operated $\mathrm{Ca}^{2+}$ entry in sensory neurons: Functional role and the effect of painful nerve injury. J. Neurosci. 2011, 31, 3536-3549. [CrossRef]

21. Bojarski, L.; Pomorski, P.; Szybinska, A.; Drab, M.; Skibinska-Kijek, A.; Gruszczynska-Biegala, J.; Kuznicki, J. Presenilin-dependent expression of STIM proteins and dysregulation of capacitative $\mathrm{Ca}^{2+}$ entry in familial Alzheimer's disease. Biochim. Biophys. Acta. 2009, 1793, 1050-1057. [CrossRef] [PubMed]

22. Wu, J.; Shih, H.-P.; Vigont, V.; Hrdlicka, L.; Diggins, L.; Singh, C.; Mahoney, M.; Chesworth, R.; Shapiro, G.; Zimina, O.; et al. Neuronal Store-Operated Calcium Entry Pathway as a Novel Therapeutic Target for Huntington's Disease Treatment. Chem. Biol. 2011, 18, 777-793. [CrossRef] [PubMed]

23. Czeredys, M.; Vigont, V.A.; Boeva, V.A.; Mikoshiba, K.; Kaznacheyeva, E.V.; Kuznicki, J. Huntingtin-Associated Protein 1A Regulates Store-Operated Calcium Entry in Medium Spiny Neurons From Transgenic YAC128 Mice, a Model of Huntington's Disease. Front. Cell. Neurosci. 2018, 12. [CrossRef]

24. Secondo, A.; Bagetta, G.; Amantea, D. On the Role of Store-Operated Calcium Entry in Acute and Chronic Neurodegenerative Diseases. Front. Mol. Neurosci. 2018, 11, 87. [CrossRef] [PubMed]

25. Erro, A.B.; Braun, A.; Kraft, R.; Kleinschnitz, C.; Schuhmann, M.K.; Stegner, D.; Wultsch, T.; Eilers, J.; Meuth, S.G.; Stoll, G.; et al. STIM2 Regulates Capacitive $\mathrm{Ca}^{2+}$ Entry in Neurons and Plays a Key Role in Hypoxic Neuronal Cell Death. Sci. Signal. 2009, 2, ra67. [CrossRef]

26. Oh-Hora, M.; Yamashita, M.; Hogan, P.G.; Sharma, S.; Lamperti, E.; Chung, W.; Prakriya, M.; Feske, S.; Rao, A. Dual functions for the endoplasmic reticulum calcium sensors STIM1 and STIM2 in T cell activation and tolerance. Nat. Immunol. 2008, 9, 432-443. [CrossRef] [PubMed]

27. Garcia-Alvarez, G.; Shetty, M.S.; Lu, B.; Yap, K.A.F.; Oh-Hora, M.; Sajikumar, S.; Bichler, Z.; Fivaz, M. Impaired spatial memory and enhanced long-term potentiation in mice with forebrain-specific ablation of the Stim genes. Front. Behav. Neurosci. 2015, 9, 180. [CrossRef] [PubMed]

28. Kalueff, A.V.; Gebhardt, M.; Stewart, A.M.; Cachat, J.M.; Brimmer, M.; Chawla, J.S.; Craddock, C.; Kyzar, E.J.; Roth, A.; Landsman, S.; et al. Towards a Comprehensive Catalog of Zebrafish Behavior 1.0 and Beyond. Zebrafish 2013, 10, 70-86. [CrossRef] [PubMed]

29. Orger, M.B.; De Polavieja, G.G. Zebrafish Behavior: Opportunities and Challenges. Annu. Rev. Neurosci. 2017, 40, 125-147. [CrossRef] [PubMed]

30. Schnörr, S.; Steenbergen, P.; Richardson, M.; Champagne, D. Measuring thigmotaxis in larval zebrafish. Behav. Brain Res. 2012, 228, 367-374. [CrossRef]

31. Lundegaard, P.R.; Anastasaki, C.; Grant, N.J.; Sillito, R.R.; Zich, J.; Zeng, Z.; Paranthaman, K.; Larsen, A.P.; Armstrong, D.; Porteous, D.J.; et al. MEK Inhibitors Reverse cAMP-Mediated Anxiety in Zebrafish. Chem. Biol. 2015, 22, 1335-1346. [CrossRef] [PubMed] 
32. Bai, Y.; Liu, H.; Huang, B.; Wagle, M.; Guo, S. Identification of environmental stressors and validation of light preference as a measure of anxiety in larval zebrafish. BMC Neurosci. 2016, 17, 63. [CrossRef] [PubMed]

33. Kedra, M.; Banasiak, K.; Kisielewska, K.; Wolinska-Niziol, L.; Jaworski, J.; Zmorzynska, J. TrkB hyperactivity contributes to brain dysconnectivity, epileptogenesis, and anxiety in zebrafish model of Tuberous Sclerosis Complex. Proc. Natl. Acad. Sci. USA 2020, 117, 2170-2179. [CrossRef]

34. Wiley, D.; Redfield, S.; Zon, L. Chemical screening in zebrafish for novel biological and therapeutic discovery. Methods Cell Biol. 2016, 138, 651-679. [CrossRef]

35. Baraban, S.C.; Dinday, M.T.; Castro, P.A.; Chege, S.; Guyenet, S.; Taylor, M.R. A large-scale mutagenesis screen to identify seizure-resistant zebrafish. Epilepsia 2007, 48, 1151-1157. [CrossRef] [PubMed]

36. Ensembl Genome Browser. Available online: www.ensembl.org (accessed on 1 April 2020).

37. Chan, C.M.; Chen, Y.; Hung, T.S.; Miller, A.; Shipley, A.M.; Webb, S. Inhibition of SOCE disrupts cytokinesis in zebrafish embryos via inhibition of cleavage furrow deepening. Int. J. Dev. Biol. 2015, 59, $289-301$. [CrossRef] [PubMed]

38. Pavez, M.; Thompson, A.C.; Arnott, H.J.; Mitchell, C.B.; D'Atri, I.; Don, E.K.; Chilton, J.K.; Scott, E.K.; Lin, J.Y.; Young, K.M.; et al. STIM1 Is Required for Remodeling of the Endoplasmic Reticulum and Microtubule Cytoskeleton in Steering Growth Cones. J. Neurosci. 2019, 39, 5095-5114. [CrossRef]

39. Motiani, R.K.; Tanwar, J.; Raja, D.A.; Vashisht, A.; Khanna, S.; Sharma, S.; Srivastava, S.; Sivasubbu, S.; Natarajan, V.T.; Gokhale, A.R.S. STIM 1 activation of adenylyl cyclase 6 connects $\mathrm{Ca}^{2+}$ and cAMP signaling during melanogenesis. EMBO J. 2018, 37, e97597. [CrossRef]

40. Ahrens, M.B.; Orger, M.B.; Robson, D.; Li, J.; Keller, P.J. Whole-brain functional imaging at cellular resolution using light-sheet microscopy. Nat. Methods 2013, 10, 413-420. [CrossRef]

41. Matthews, M.; Trevarrow, B.; Matthews, J. A virtual tour of the Guide for zebrafish users. Lab Anim. 2002, 31, 34-40.

42. Liu, Y.; Carmer, R.; Zhang, G.; Venkatraman, P.; Brown, S.A.; Pang, C.-P.; Zhang, M.; Ma, P.; Leung, Y.F. Statistical Analysis of Zebrafish Locomotor Response. PLoS ONE 2015, 10, e0139521. [CrossRef] [PubMed]

43. Knafo, S.; Fidelin, K.; Prendergast, A.; Tseng, P.-E.B.; Parrin, A.; Dickey, C.W.; Böhm, U.L.; Figueiredo, S.N.; Thouvenin, O.; Pascal-Moussellard, H.; et al. Mechanosensory neurons control the timing of spinal microcircuit selection during locomotion. eLife 2017, 6, 6. [CrossRef]

44. Panier, T.; Romano, S.; Olive, R.; Pietri, T.; Sumbre, G.; Candelier, R.; Debrégeas, G. Fast functional imaging of multiple brain regions in intact zebrafish larvae using selective plane illumination microscopy. Front. Neural Circuit. 2013, 7, 65. [CrossRef] [PubMed]

45. Peterson, S.M.; Freeman, J.L. RNA Isolation from Embryonic Zebrafish and cDNA Synthesis for Gene Expression Analysis. J. Vis. Exp. 2009. [CrossRef] [PubMed]

46. Burgess, H.A.; Granato, M. Modulation of locomotor activity in larval zebrafish during light adaptation. J. Exp. Biol. 2007, 210, 2526-2539. [CrossRef]

47. Meldrum, B.S. The role of glutamate in epilepsy and other CNS disorders. Neurology 1994, 44, 14-23.

48. Gruszczynska-Biegala, J.; Sladowska, M.; Kuznicki, J. AMPA Receptors Are Involved in Store-Operated Calcium Entry and Interact with STIM Proteins in Rat Primary Cortical Neurons. Front. Cell. Neurosci. 2016, 10, 1289. [CrossRef]

49. Gruszczynska-Biegala, J.; Strucinska, K.; Maciag, F.; Majewski, L.; Sladowska, M.; Kuznicki, J. STIM Protein-NMDA2 Receptor Interaction Decreases NMDA-Dependent Calcium Levels in Cortical Neurons. Cells 2020, 9, 160. [CrossRef]

50. Zhou, S.; Li, C.-S.; Liu, L.-Q.; Li, Y.; Wang, X.-F.; Shen, L. Increased expression of annexin A7 in temporal lobe tissue of patients with refractory epilepsy. Histol. Histopathol. 2011, 26, 571-579.

51. Zub, E.; Canet, G.; Garbelli, R.; Blaquiere, M.; Rossini, L.; Pastori, C.; Sheikh, M.; Reutelingsperger, C.; Klement, W.; De Bock, F.; et al. The GR-ANXA1 pathway is a pathological player and a candidate target in epilepsy. FASEB J. 2019, 33, 13998-14009. [CrossRef]

52. Chong, K.W.Y.; Chen, M.J.; Koay, E.S.; Wong, B.-S.; Lee, A.Y.-W.; Russo-Marie, F.; Cheung, N.S. Annexin A3 is associated with cell death in lactacystin-mediated neuronal injury. Neurosci. Lett. 2010, 485, 129-133. [CrossRef] [PubMed]

53. Simats, A.; García-Berrocoso, T.; Montaner, J. Neuroinflammatory biomarkers: From stroke diagnosis and prognosis to therapy. Biochim. Biophys. Acta 2016, 1862, 411-424. [CrossRef] [PubMed] 
54. Gonzalez, A.M.; Podvin, S.; Lin, S.; Miller, M.C.; Botfield, H.; E Leadbeater, W.; Roberton, A.; Dang, X.; E Knowling, S.; Cardenas-Galindo, E.; et al. Ecrg4 expression and its product augurin in the choroid plexus: Impact on fetal brain development, cerebrospinal fluid homeostasis and neuroprogenitor cell response to CNS injury. Fluids Barriers CNS 2011, 8, 6. [CrossRef] [PubMed]

55. Kujuro, Y.; Suzuki, N.; Kondo, T. Esophageal cancer-related gene 4 is a secreted inducer of cell senescence expressed by aged CNS precursor cells. Proc. Natl. Acad. Sci. USA 2010, 107, 8259-8264. [CrossRef] [PubMed]

56. Hwang, D.Y.; Woo, J.-M.; Park, S.J.; Kang, H.I.; Kim, B.G.; Shim, S.B.; Jee, S.W.; Lee, S.H.; Sin, J.S.; Bae, C.J.; et al. Characterization of changes in global gene expression in the brain of neuron-specific enolase/human Tau23 transgenic mice in response to overexpression of Tau protein. Int. J. Mol. Med. 2010, 25, 667-675. [CrossRef]

57. Hershfinkel, M. The Zinc Sensing Receptor, ZnR/GPR39, in Health and Disease. Int. J. Mol. Sci. 2018, 19, 439. [CrossRef]

58. Alavi, M.S.; Shamsizadeh, A.; Azhdari-Zarmehri, H.; Roohbakhsh, A. Orphan G protein-coupled receptors: The role in CNS disorders. Biomed. Pharmacother. 2018, 98, 222-232. [CrossRef]

59. Fazio, G.; Gaston-Massuet, C.; Bettini, L.R.; Graziola, F.; Scagliotti, V.; Cereda, A.; Ferrari, L.; Mazzola, M.; Cazzaniga, G.; Giordano, A.; et al. CyclinD1 Down-Regulation and Increased Apoptosis Are Common Features of Cohesinopathies. J. Cell. Physiol. 2015, 231, 613-622. [CrossRef]

60. Goldstein, J.H.; Tim-Aroon, T.; Shieh, J.; Merrill, M.; Deeb, K.K.; Zhang, S.; Bass, N.E.; Bedoyan, J.K. Novel SMC1A frameshift mutations in children with developmental delay and epilepsy. Eur. J. Med Genet. 2015, 58, 562-568. [CrossRef]

61. Lebrun, N.; Lebon, S.; Jeannet, P.-Y.; Jacquemont, S.; Billuart, P.; Bienvenu, T. Early-onset encephalopathy with epilepsy associated with a novel splice site mutation inSMC1A. Am. J. Med Genet. Part A 2015, 167, 3076-3081. [CrossRef]

62. Barresi, M.J.; Burton, S.; Dipietrantonio, K.; Amsterdam, A.; Hopkins, N.; Karlstrom, R.O. Essential genes for astroglial development and axon pathfinding during zebrafish embryogenesis. Dev. Dyn. 2010, 239, 2603-2618. [CrossRef] [PubMed]

63. Udagawa, T.; Swanger, S.A.; Takeuchi, K.; Kim, J.H.; Nalavadi, V.; Shin, J.; Lorenz, L.J.; Zukin, R.S.; Bassell, G.J.; Richter, J.D. Bidirectional control of mRNA translation and synaptic plasticity by the cytoplasmic polyadenylation complex. Mol. Cell 2012, 47, 253-266. [CrossRef] [PubMed]

64. Huang, J.; Zhong, Z.; Wang, M.; Chen, X.; Tan, Y.; Zhang, S.; He, W.; He, X.; Huang, G.; Lu, H.; et al. Circadian Modulation of Dopamine Levels and Dopaminergic Neuron Development Contributes to Attention Deficiency and Hyperactive Behavior. J. Neurosci. 2015, 35, 2572-2587. [CrossRef]

65. Shiraishi-Yamaguchi, Y.; Sato, Y.; Sakai, R.; Mizutani, A.; Knöpfel, T.; Mori, N.; Mikoshiba, K.; Furuichi, T. Interaction of Cupidin/Homer2 with two actin cytoskeletal regulators, Cdc42 small GTPase and Drebrin, in dendritic spines. BMC Neurosci. 2009, 10, 25. [CrossRef] [PubMed]

66. Shin, D.M.; Dehoff, M.; Luo, X.; Kang, S.H.; Tu, J.; Nayak, S.K.; Ross, E.M.; Worley, P.; Muallem, S. Homer 2 tunes $\mathrm{G}$ protein-coupled receptors stimulus intensity by regulating RGS proteins and PLC $\beta$ GAP activities. J. Cell Biol. 2003, 162, 293-303. [CrossRef] [PubMed]

67. Jardin, I.; Lopez, J.J.; Erro, A.B.; Salido, G.M.; Rosado, J.A. Homer Proteins in Ca ${ }^{2+}$ Entry. IUBMB Life 2013, 65, 497-504. [CrossRef] [PubMed]

68. Yang, Y.-M.; Lee, J.; Jo, H.; Park, S.; Chang, I.; Muallem, S.; Shin, D.M. Homer2 Protein Regulates Plasma Membrane $\mathrm{Ca}^{2+}$-ATPase-mediated $\mathrm{Ca}^{2+}$ Signaling in Mouse Parotid Gland Acinar Cells. J. Biol. Chem. 2014, 289, 24971-24979. [CrossRef] [PubMed]

69. Majewski, L.; Maciag,, F.; Boguszewski, P.M.; Kuznicki, J. Transgenic Mice Overexpressing Human STIM2 and ORAI1 in Neurons Exhibit Changes in Behavior and Calcium Homeostasis but Show No Signs of Neurodegeneration. Int. J. Mol. Sci. 2020, 21, 842. [CrossRef] [PubMed]

70. Yang, L.; Chang, S.; Lu, Q.; Zhang, Y.; Wu, Z.; Sun, X.; Cao, Q.; Qian, Y.; Jia, T.; Xu, B.; et al. A new locus regulating MICALL2 expression was identified for association with executive inhibition in children with attention deficit hyperactivity disorder. Mol. Psychiatry 2017, 23, 1014-1020. [CrossRef]

71. Lange, M.; Norton, W.H.; Coolen, M.; Chaminade, M.; Merker, S.; Proft, F.; Schmitt, A.; Vernier, P.; Lesch, K.-P.; Bally-Cuif, L. The ADHD-susceptibility gene lphn3.1 modulates dopaminergic neuron formation and locomotor activity during zebrafish development. Mol. Psychiatry 2012, 17, 946-954. [CrossRef] 
72. Taylor, A.; Steinberg, J.; Webber, C. Duplications in ADHD patients harbour neurobehavioural genes that are co-expressed with genes associated with hyperactivity in the mouse. Am. J. Med Genet. 2015, 168, 97-107. [CrossRef] [PubMed]

73. De Calbiac, H.; Dabacan, A.; Marsan, E.; Tostivint, H.; Devienne, G.; Ishida, S.; LeGuern, E.; Baulac, S.; Muresan, R.C.; Kabashi, E.; et al. Depdc5 knockdown causes mTOR-dependent motor hyperactivity in zebrafish. Ann. Clin. Transl. Neurol. 2018, 5, 510-523. [CrossRef] [PubMed]

74. Baraban, S.C.; Taylor, M.; Castro, P.; Baier, H. Pentylenetetrazole induced changes in zebrafish behavior, neural activity and c-fos expression. Neuroscience 2005, 131, 759-768. [CrossRef] [PubMed]

75. McCutcheon, V.; Park, E.; Liu, E.; Wang, Y.; Wen, X.-Y.; Baker, A.J. A Model of Excitotoxic Brain Injury in Larval Zebrafish: Potential Application for High-Throughput Drug Evaluation to Treat Traumatic Brain Injury. Zebrafish 2016, 13, 161-169. [CrossRef] [PubMed]

(C) 2020 by the authors. Licensee MDPI, Basel, Switzerland. This article is an open access article distributed under the terms and conditions of the Creative Commons Attribution (CC BY) license (http://creativecommons.org/licenses/by/4.0/). 